\title{
Conjugations in $L^{2}(\mathcal{H})$
}

\author{
M. Cristina Câmara, Kamila Kliś-Garlicka®, Bartosz Łanucha® and \\ Marek Ptak
}

\begin{abstract}
Conjugations commuting with $\mathbf{M}_{z}$ and intertwining $\mathbf{M}_{z}$ and $\mathbf{M}_{\bar{z}}$ in $L^{2}(\mathcal{H})$, where $\mathcal{H}$ is a separable Hilbert space, are characterized. We also investigate which of them leave invariant the whole Hardy space $H^{2}(\mathcal{H})$ or a model space $K_{\Theta}=H^{2}(\mathcal{H}) \ominus \Theta H^{2}(\mathcal{H})$, where $\Theta$ is a pure operator valued inner function.
\end{abstract}

Mathematics Subject Classification. Primary 47B35; Secondary 47B32, $30 \mathrm{D} 20$.

Keywords. Conjugation, $C$-symmetric operator, Hardy space, Model space, Invariant subspaces for unilateral shift, Model for a contraction, Truncated Toeplitz operator.

\section{Introduction}

The motivation to study conjugations (i.e., antilinear isometric involutions) has its roots in physics ([8]), in particular in non-hermitian quantum mechanics and spectral analysis of complex symmetric operators. There are many important examples of complex symmetric operators, that is $C$-symmetric operators with respect to some conjugation $C$, namely normal operators, Hankel operators, truncated Toeplitz operators (see for example [3-9, 11, 14]).

In $[2,3]$ all conjugations in the classical $L^{2}$ space on the unit circle commuting with $M_{z}$ or intertwining the operators $M_{z}$ and $M_{\bar{z}}$ (in other words, all conjugations $C$ according to which the operator $M_{z}$ is $C$-symmetric, see the definition below) were fully characterized. The behaviour of such conjugations was also studied in connection with an analytic part of the space $L^{2}$ and model spaces, in particular there were characterized all conjugations leaving the whole Hardy space and model spaces invariant. In what follows we study similar questions concerning conjugations in $L^{2}$ spaces with values in a

The work of the first author was partially supported by FCT/Portugal through UID/MAT/04459/2019. The research of the second and the fourth authors was financed by the Ministry of Science and Higher Education of the Republic of Poland. 
certain Hilbert space $\mathcal{H}$. The investigation in this direction is important for its relation with Sz.-Nagy-Foiaş theory [12, Chap. 6] saying that $C_{0}$ contractions with finite defect indexes are unitarily equivalent to multiplication by the independent variable in a certain model space given by an operator valued inner function. In other words, the results from the paper can be moved by unitary equivalence to contractions on Hilbert spaces, keeping suitable assumptions.

Denote by $\mathcal{H}$ a complex Hilbert space, by $L(\mathcal{H})$ the algebra of all bounded linear operators on $\mathcal{H}$ and by $L A(\mathcal{H})$ the space of all bounded antilinear operators on $\mathcal{H}$. A conjugation $C$ in $\mathcal{H}$ is an antilinear isometric involution, i.e., $C^{2}=I_{\mathcal{H}}$ and

$$
\langle C f, C g\rangle=\langle g, f\rangle \quad \text { for all } f, g \in \mathcal{H} .
$$

An operator $A \in L(\mathcal{H})$ is called $C$-symmetric if $C A C=A^{*}$. Recall that for $A \in L A(\mathcal{H})$ there exists a unique antilinear operator $A^{\sharp}$, called the antilinear adjoint of $A$, defined by the equality

$$
\langle A f, g\rangle=\overline{\left\langle f, A^{\sharp} g\right\rangle},
$$

for all $f, g \in \mathcal{H}$. It is clear, see [3], that $C^{\sharp}=C$ for any conjugation $C$, $(A B)^{\sharp}=B^{*} A^{\sharp}$ and similarly $(B A)^{\sharp}=A^{\sharp} B^{*}$ for $A \in L A(\mathcal{H}), B \in L(\mathcal{H})$.

Let $L^{2}=L^{2}(\mathbb{T}, m)$ and $L^{\infty}=L^{\infty}(\mathbb{T}, m)$ where $\mathbb{T}$ is the unit circle and $m$ is the normalized Lebesgue measure and let $H^{2}$ denote the classical Hardy space on the unit disc $\mathbb{D}$. For an inner function $\theta$ (i.e., $\theta \in L^{\infty} \cap H^{2}$ and $|\theta|=1$ a.e. on $\mathbb{T})$ one can define the model space $K_{\theta}=H^{2} \ominus \theta H^{2}$.

The most natural conjugation $\tilde{J}$ in $L^{2}$ is defined as $\tilde{J} f=\bar{f}$, for $f \in L^{2}$. This conjugation has two natural properties: the operator $M_{z}$ is $\tilde{J}_{\text {-symmetric, }}$ i.e., $M_{z} \tilde{J}=\tilde{J} M_{\bar{z}}$, and $\tilde{J}$ maps an analytic function into a co-analytic one, i.e., $\tilde{J} H^{2}=\overline{H^{2}}$. Another natural conjugation in $L^{2}$ is $J^{\star} f=f^{\#}, f^{\#}(z)=\overline{f(\bar{z})}$. The conjugation $J^{\star}$ has a completely different behaviour: it commutes with multiplication by $z\left(M_{z} J^{\star}=J^{\star} M_{z}\right)$ and leaves analytic functions invariant, $J^{\star} H^{2}=H^{2}$.

In Sect. 3 we recall some basic properties of vector and operator valued functions. In Sect. 4, for any separable Hilbert space $\mathcal{H}$, we naturally extend the definitions of the conjugations $\tilde{J}$ and $J^{\star}$ on $L^{2}$ to the conjugations $\widetilde{\mathbf{J}}$ and $\mathbf{J}^{\star}$ on a vector valued space $L^{2}(\mathcal{H})$ keeping the same properties with respect to the multiplication by the independent variable, i.e., $\widetilde{\mathbf{J}} \mathbf{M}_{z}=\mathbf{M}_{\bar{z}} \widetilde{\mathbf{J}}, \quad \mathbf{J}^{\star} \mathbf{M}_{z}=$ $\mathbf{M}_{z} \mathbf{J}^{\star}$. However, one needs to fix some conjugation $J$ on $\mathcal{H}$ (in case of $L^{2}$ the natural conjugation in $\mathbb{C}, z \mapsto \bar{z}$, plays this role). Theorems 4.8 and 4.3

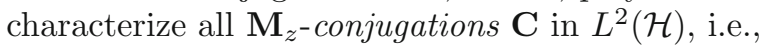

$$
\mathrm{CM}_{z}=\mathbf{M}_{\bar{z}} \mathbf{C}
$$

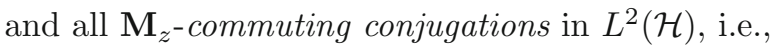

$$
\mathrm{CM}_{z}=\mathbf{M}_{z} \mathbf{C} \text {. }
$$

In Sect. 5, Theorem 5.1 and Proposition 5.5, we describe all conjugations satisfying (1.3) or (1.4) and leaving the Hardy space $H^{2}(\mathcal{H})$ invariant. In Sect. 6 we study these conjugations for which vector valued model spaces are 
invariant. We concentrate on the case when the dimension of the underlying Hilbert space $\mathcal{H}$ is finite and the vector valued inner function is pure. The last section is devoted to those conjugations which leave shift invariant subspaces invariant. Generally, the vector valued case is much more complicated than the scalar one, see for example Theorems 6.6, 7.3, 8.6 and Example 8.8. Section 2 is devoted to the special case $\mathcal{H}=\mathbb{C}^{2}$. This illustrates the general theorems and gives stronger results than in [11]. On the other hand, it gives more precise characterizations (Theorem 2.5), which are of independent interest.

\section{Conjugations in $L^{2} \oplus L^{2}$}

In Theorem 2.4 and Proposition 2.6 [11] there are given the conditions for a $2 \times 2$ operator matrix to be a conjugation. We will, however, use equivalent conditions obtained by checking the antilinear selfadjointness and involutive property of a conjugation. Namely, let $\mathbf{C}=\left[\begin{array}{cc}D_{1} & D_{2} \\ D_{3} & D_{4}\end{array}\right]$, where $D_{j}$ are antilinear operators on $\mathcal{H}$ for $j=1,2,3,4$. Then $\mathbf{C}$ is a conjugation on $\mathcal{H} \oplus \mathcal{H}$ if and only if the following conditions hold:

$$
\begin{aligned}
& D_{1}=D_{1}^{\sharp}, D_{4}=D_{4}^{\sharp}, D_{3}=D_{2}^{\sharp}, \\
& D_{1} D_{1}^{\sharp}+D_{2} D_{2}^{\sharp}=I, D_{2}^{\sharp} D_{2}+D_{4} D_{4}^{\sharp}=I, \\
& D_{2}^{\sharp} D_{1}+D_{4} D_{2}^{\sharp}=0 .
\end{aligned}
$$

Denote $\mathbf{M}_{z}=\left[\begin{array}{cc}M_{z} & 0 \\ 0 & M_{z}\end{array}\right]$. We will investigate the conditions for $\mathbf{C}$ to be an $\mathbf{M}_{z}$-conjugation and for it to commute with $\mathbf{M}_{z}$.

In [2, Theorem 2.4] all conjugations in $L^{2}$ commuting with $M_{z}$ were characterized. In particular it was shown that such a conjugation has to be of the form $M_{\psi} J^{\star}$ for some unimodular function $\psi \in L^{\infty}$ which is symmetric, i.e., $\psi(z)=\psi(\bar{z})$ a.e. on $\mathbb{T}$. The following theorem gives a characterization of $\mathbf{M}_{z}$-commuting conjugations in $L^{2} \oplus L^{2}$.

Theorem 2.1. Let $\mathbf{C}$ be an antilinear operator on $L^{2} \oplus L^{2}$. Then $\mathbf{C}=\left[\begin{array}{ll}D_{1} & D_{2} \\ D_{2}^{\sharp} & D_{4}\end{array}\right]$ is a conjugation such that $\mathbf{M}_{z} \mathbf{C}=\mathbf{C M}_{z}$ if and only if there are functions $\psi_{i} \in L^{\infty}, i=1,2,4$, such that $D_{i}=M_{\psi_{i}} J^{\star}$ and

$$
\begin{aligned}
& \psi_{1}^{\#}=\bar{\psi}_{1}, \quad \psi_{4}^{\#}=\bar{\psi}_{4}, \\
& \left|\psi_{1}\right|^{2}=\left|\psi_{4}\right|^{2}=1-\left|\psi_{2}\right|^{2}, \\
& \psi_{1}^{\#} \psi_{2}+\psi_{2}^{\#} \psi_{4}=0 .
\end{aligned}
$$

Proof. Easy calculations show that $\mathbf{M}_{z} \mathbf{C}=\mathbf{C M}_{z}$ if and only if $M_{z} D_{i}$ $=D_{i} M_{z}$ for $i=1,2,4$ and $M_{z} D_{2}^{\sharp}=D_{2}^{\sharp} M_{z}$. Hence for $i=1,2,4$ we have

$$
M_{z} D_{i} J^{\star}=D_{i} M_{z} J^{\star}=D_{i} J^{\star} M_{z} .
$$

Thus the linear operators $D_{i} J^{\star}$ commute with $M_{z}$, so they have to be of the form $D_{i} J^{\star}=M_{\psi_{i}}$ for $\psi_{i} \in L^{\infty}$. 
Condition (2.1) implies that for $i=1,4, M_{\psi_{i}} J^{\star}=J^{\star} M_{\bar{\psi}_{i}}$, i.e., $\psi_{i}^{\#}=\bar{\psi}_{i}$. This means that $\psi_{1}$ and $\psi_{4}$ are symmetric, i.e., $\psi_{i}(z)=\psi_{i}(\bar{z})$ for $i=1,4$ (a.e. on $\mathbb{T}$ ). By $(2.2)$, for $i=1,4$, we get

$$
\left(M_{\psi_{i}} J^{\star}\right)^{2}+M_{\psi_{2}} J^{\star} J^{\star} M_{\bar{\psi}_{2}}=I
$$

which is equivalent to

$$
\psi_{i}^{\#} \psi_{i}+\left|\psi_{2}\right|^{2}=1
$$

Finally, by (2.3) we get

$$
J^{\star} M_{\bar{\psi}_{2}} M_{\psi_{1}} J^{\star}+J^{\star} M_{\psi_{4}^{\#}} M_{\bar{\psi}_{2}^{\#}} J^{\star}=0
$$

which is equivalent to

$$
\bar{\psi}_{2} \psi_{1}+\psi_{4}^{\#} \bar{\psi}_{2}^{\#}=0
$$

Taking into consideration the fact that $\psi_{1}$ and $\psi_{4}$ are symmetric we obtain (2.6).

Remark 2.2. Note that if $D_{2}=0$, then conditions (2.4)-(2.6) imply that $D_{1}$ and $D_{4}$ are conjugations which commute with $M_{z}$. Hence by $[2$, Theorem 2.4] we get $D_{1}=M_{\psi_{1}} J^{\star}, D_{4}=M_{\psi_{4}} J^{\star}$. On the other hand, this is also a consequence of Theorem 2.1.

If now $\psi_{1}=0$ (which is equivalent to $\psi_{4}=0$ ), then $(2.5)$ implies that $\left|\psi_{2}\right|=1$.

Example 2.3. The following conjugations satisfy the conditions of Theorem 2.1:

$$
\mathbf{J}_{1}^{\star}=\left[\begin{array}{cc}
J^{\star} & 0 \\
0 & J^{\star}
\end{array}\right], \quad \text { or } \quad \mathbf{J}_{2}^{\star}=\left[\begin{array}{cc}
0 & J^{\star} \\
J^{\star} & 0
\end{array}\right] \quad \text { or } \quad \mathbf{C}=\frac{1}{\sqrt{2}}\left[\begin{array}{cc}
J^{\star} & J^{\star} \\
J^{\star} & -J^{\star}
\end{array}\right] .
$$

Example 2.4. Let $\psi_{1}(z)=\psi_{4}(z)=\frac{1}{2}(\bar{z}+z)$ and $\psi_{2}(z)=\frac{1}{2 i}(-\bar{z}+z)$. In other words, $\psi_{1}\left(e^{i t}\right)=\psi_{4}\left(e^{i t}\right)=\cos t$ and $\psi_{2}\left(e^{i t}\right)=\sin t$. Then $\psi_{2}^{\#}(z)=\frac{1}{2 i}(\bar{z}-z)$, i.e., $\psi_{2}^{\#}\left(e^{i t}\right)=-\sin t$. Observe that such functions satisfy conditions (2.4)(2.6), so $\mathbf{C}=\left[\begin{array}{ll}M_{\psi_{1}} J^{\star} & M_{\psi_{2}} J^{\star} \\ M_{\bar{\psi}_{2}^{\#}}^{\#} J^{\star} & M_{\psi_{4}} J^{\star}\end{array}\right]$ is a conjugation satisfying Theorem 2.1.

The characterization of all $M_{z}$-conjugations in $L^{2}$ was given in [3]. It was proved that such conjugations are of the form $M_{\psi} \tilde{J}$, where $\psi \in L^{\infty}$, $|\psi|=1$ a.e. on $\mathbb{T}$. In the space $L^{2}\left(\mathbb{C}^{2}\right)$ the characterization is more complex.

Theorem 2.5. Let $\mathbf{C}$ be an antilinear operator on $L^{2}\left(\mathbb{C}^{2}\right)$. Then $\mathbf{C}=$ $\left[\begin{array}{cc}D_{1} & D_{2} \\ D_{2}^{\sharp} & D_{4}\end{array}\right]$ is a conjugation such that $\mathbf{M}_{z} \mathbf{C}=\mathbf{C M}_{\bar{z}}$ if and only if there are functions $\psi_{i} \in L^{\infty}, i=1,2,4$, such that $D_{i}=M_{\psi_{i}} \tilde{J}$ and

$$
\begin{aligned}
& \left|\psi_{1}\right|^{2}=\left|\psi_{4}\right|^{2}=1-\left|\psi_{2}\right|^{2}, \\
& \bar{\psi}_{1} \psi_{2}+\bar{\psi}_{2} \psi_{4}=0 .
\end{aligned}
$$


Proof. Note that $\mathbf{M}_{z} \mathbf{C}=\mathbf{C M}_{\bar{z}}$ if and only if

$$
M_{z} D_{i}=D_{i} M_{\bar{z}} \text { for } i=1,2,4 .
$$

Hence for $i=1,2,4$ we have

$$
M_{z} D_{i} \tilde{J}=D_{i} M_{\bar{z}} \tilde{J}=D_{i} \tilde{J} M_{z} .
$$

Thus the linear operators $D_{i} \tilde{J}$ commute with $M_{z}$, so they have to be of the form $D_{i} \tilde{J}=M_{\psi_{i}}$ for $\psi_{i} \in L^{\infty}$. Hence $D_{i}=M_{\psi_{i}} \tilde{J}$ for $\psi_{i} \in L^{\infty}$.

Note that $\mathbf{C}$ has to satisfy conditions (2.1)-(2.3). Condition (2.1) is satisfied automatically since $D_{i}=M_{\psi_{i}} \tilde{J}$ are antilinearly self-adjoint for $\psi_{i} \in$ $L^{\infty}$. By checking (2.2) we get

$$
\left(M_{\psi_{1}} \tilde{J}\right)^{2}+M_{\psi_{2}} \tilde{J} \tilde{J} M_{\bar{\psi}_{2}}=I \text { and }\left(M_{\psi_{4}} \tilde{J}\right)^{2}+M_{\psi_{2}} \tilde{J} \tilde{J} M_{\bar{\psi}_{2}}=I,
$$

which is equivalent to (2.9). Finally by $(2.3)$ we get

$$
\tilde{J} M_{\bar{\psi}_{2}} M_{\psi_{1}} \tilde{J}+M_{\psi_{4}} \tilde{J} \tilde{J} M_{\bar{\psi}_{2}}=0
$$

which is equivalent to $(2.10)$.

Remark 2.6. Note that if (2.9)-(2.10) are satisfied and $\psi_{2}=0$, then $\left|\psi_{1}\right|=$ $\left|\psi_{4}\right|=1$. Hence $D_{1}$ and $D_{4}$ are $M_{z}$-conjugations in $L^{2}$. On the other hand, if $\psi_{1}=0$ (which is equivalent to $\psi_{4}=0$ ), then $\left|\psi_{2}\right|=1$, which implies that $D_{2}$ is an $M_{z}$-conjugation.

Example 2.7. The following conjugations satisfy Theorem 2.5:

$$
\widetilde{\mathbf{J}}_{1}=\left[\begin{array}{cc}
\tilde{J} & 0 \\
0 & \tilde{J}
\end{array}\right], \quad \text { or } \quad \widetilde{\mathbf{J}}_{2}=\left[\begin{array}{cc}
0 & \tilde{J} \\
\tilde{J} & 0
\end{array}\right], \quad \text { or } \quad \mathbf{C}=\frac{1}{\sqrt{2}}\left[\begin{array}{cc}
\tilde{J} & \tilde{J} \\
\tilde{J} & -\tilde{J}
\end{array}\right] .
$$

Example 2.8. Let $\psi_{1}(z)=-\psi_{4}(z)=\frac{1}{2 i}(-\bar{z}+z)$ and $\psi_{2}(z)=\frac{1}{2}(\bar{z}+z)$. In other words, $\psi_{1}\left(e^{i t}\right)=-\psi_{4}\left(e^{i t}\right)=\sin t$ and $\psi_{2}\left(e^{i t}\right)=\cos t$. Observe that

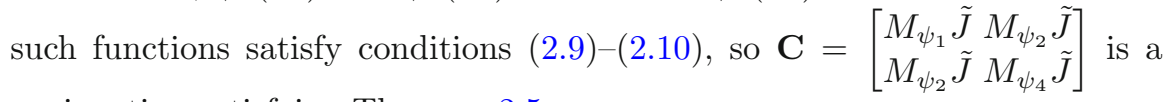
conjugation satisfying Theorem 2.5 .

\section{Operator Valued Functions}

Let $\mathcal{H}$ be a complex separable Hilbert space. Denote by $L^{2}(\mathcal{H})$ the space of all (classes of) functions $\mathbf{f}: \mathbb{T} \rightarrow \mathcal{H}$ which are measurable and satisfy

$$
\int_{\mathbb{T}}\|\mathbf{f}(z)\|^{2} d m(z)<\infty
$$

(the norm $\|\cdot\|$ under the integral is the norm in $\mathcal{H}$ ). So $\mathbf{f} \in L^{2}(\mathcal{H})$ is understood as a class (represented by $\mathbf{f}$ ) of all measurable functions satisfying (3.1) and equal to $\mathbf{f}$ on $\mathbb{T}$ a.e. with respect to $m$. Recall that the measurability of f means that $z \mapsto\|\mathbf{f}(z)\|$ is a measurable function (or that $z \mapsto\langle\mathbf{f}(z), x\rangle$ is measurable for every $x \in \mathcal{H}$, which due to separability of $\mathcal{H}$ is an equivalent definition). 
The space $L^{2}(\mathcal{H})$ is a Hilbert space with the inner product given by

$$
\langle\mathbf{f}, \mathbf{g}\rangle=\int_{\mathbb{T}}\langle\mathbf{f}(z), \mathbf{g}(z)\rangle d m(z), \quad \mathbf{f}, \mathbf{g} \in L^{2}(\mathcal{H}),
$$

where the inner product under the integral is the inner product in $\mathcal{H}$ (note that $L^{2}(\mathcal{H})$ is also separable).

Functions in $L^{2}(\mathcal{H})$ have an expansion analogous to the Fourier expansion in $L^{2}$. Observe that if $\mathbf{f} \in L^{2}(\mathcal{H})$, then for each $n \in \mathbb{Z}$ the linear functional $y \mapsto \overline{\int_{\mathbb{T}}\langle\mathbf{f}(z), y\rangle \bar{z}^{n} d m(z)}$ is bounded and so there exists $x_{n} \in \mathcal{H}$ such that

$$
\left\langle x_{n}, y\right\rangle=\int_{\mathbb{T}}\langle\mathbf{f}(z), y\rangle \bar{z}^{n} d m(z) \quad \text { for all } y \in \mathcal{H} .
$$

The element $x_{n}$ is called the $n$-th Fourier coefficient of $\mathbf{f}$. It turns out that $\mathbf{f} \in L^{2}(\mathcal{H})$ can be expressed as $\mathbf{f}=\sum_{n=-\infty}^{\infty} x_{n} e_{n}$, where $x_{n}$ is given by (3.2), $e_{n}(z)=z^{n}$ for $z \in \mathbb{T}$ and the series converges in the norm of $L^{2}(\mathcal{H})$. Moreover, for $\mathbf{f}=\sum_{n=-\infty}^{\infty} x_{n} e_{n} \in L^{2}(\mathcal{H})$ and $\mathbf{g}=\sum_{n=-\infty}^{\infty} y_{n} e_{n} \in L^{2}(\mathcal{H})$ we have

$$
\|\mathbf{f}\|^{2}=\sum_{n=-\infty}^{\infty}\left\|x_{n}\right\|^{2}
$$

and

$$
\langle\mathbf{f}, \mathbf{g}\rangle=\sum_{n=-\infty}^{\infty}\left\langle x_{n}, y_{n}\right\rangle,
$$

and this correspondence between elements of $L^{2}(\mathcal{H})$ and sequences $\left\{x_{n}\right\}_{n=-\infty}^{\infty} \subset \mathcal{H}$ such that $\sum_{n=-\infty}^{\infty}\left\|x_{n}\right\|^{2}<\infty$ is one-to-one (see, e.g., [13, pp. 46-48]).

Denote by $H^{2}(\mathcal{H})$ the subspace of $L^{2}(\mathcal{H})$ consisting of those functions from $L^{2}(\mathcal{H})$ whose Fourier coefficients with negative indices are 0, i.e.,

$$
H^{2}(\mathcal{H})=\left\{\mathbf{f} \in L^{2}(\mathcal{H}): \mathbf{f}=\sum_{n=0}^{\infty} x_{n} e_{n}\right\} .
$$

Each $\mathbf{f} \in H^{2}(\mathcal{H})$ can be also identified with a function

$$
\mathbf{f}(\lambda)=\sum_{n=0}^{\infty} x_{n} \lambda^{n}, \quad \lambda \in \mathbb{D},
$$

which is analytic in the unit disk $\mathbb{D}$. Thus $H^{2}(\mathcal{H})$ can be seen as a subspace of $L^{2}(\mathcal{H})$ or as a space of functions analytic in $\mathbb{D}$ and with values in $\mathcal{H}$. The boundary values on $\mathbb{T}$ can be then obtained through radial limits (here the radial functions converge to the boundary function in the $L^{2}(\mathcal{H})$ norm). For more details see also $[1,12]$.

Now let us recall after [1] some notations. Let $H^{\infty}(L(\mathcal{H}))$ denote the set of bounded $L(\mathcal{H})$-valued analytic functions, $\mathbf{F}: \mathbb{D} \rightarrow L(\mathcal{H})$ with the norm $\|\mathbf{F}\|_{\infty}=\sup \{\|\mathbf{F}(\lambda)\|:|\lambda|<1\}$. On the other hand, we can consider functions on the unit circle. A function $\mathbf{F}: \mathbb{T} \rightarrow L(\mathcal{H})$ is said to be measurable, if 
for every $x \in \mathcal{H}$ the function $z \mapsto \mathbf{F}(z) x$ is measurable. Let us denote by $L^{\infty}(L(\mathcal{H}))$ the space of (again, classes of) all such measurable functions $\mathbf{F}$ which are essentially bounded, i.e, $\|\mathbf{F}\|_{\infty}=\operatorname{ess}_{\sup _{z \in \mathbb{T}}}\|\mathbf{F}(z)\|<\infty$. Every $\mathbf{F} \in L^{\infty}(L(\mathcal{H}))$ admits a Fourier expansion given by the formal series

$$
\mathbf{F}=\sum_{n=-\infty}^{\infty} F_{n} e_{n},
$$

with $F_{n} \in L(\mathcal{H})$ for $n \in \mathbb{Z}$. Each of the coefficients $F_{n}$ is defined as an integral in the strong sense, i.e.,

$$
F_{n} h=\int_{\mathbb{T}} z^{-n} \mathbf{F}(z) h d m(z) \quad \text { for } \quad h \in \mathcal{H} .
$$

The space $H^{\infty}(L(\mathcal{H}))$ can be identified with the subspace of $L^{\infty}(L(\mathcal{H}))$ consisting of those functions $\mathbf{F}$ whose Fourier coefficients $F_{n}$ vanish if $n<0$. Namely, every bounded analytic function from $H^{\infty}(L(\mathcal{H}))$ has boundary values a.e. on $\mathbb{T}$, the boundary function belongs to $L^{\infty}(L(\mathcal{H}))$ and its Fourier coefficients with negative indices vanish. Conversely, every $\mathbf{F}=\sum_{n=0}^{\infty} F_{n} e_{n} \in$ $L^{\infty}(L(\mathcal{H}))$ can be then extended to a function analytic in $\mathbb{D}$ by the formula

$$
\mathbf{F}(\lambda)=\sum_{n=0}^{\infty} F_{n} \lambda^{n}, \quad \lambda \in \mathbb{D},
$$

and this extension is bounded. Moreover, both $\|\cdot\|_{\infty}$ coincide. Therefore a function from $H^{\infty}(L(\mathcal{H}))$ can be seen as an element of $L^{\infty}(L(\mathcal{H}))$ or as a bounded analytic operator valued function in $\mathbb{D}$ (see [1, p. 232]). Note that for $\mathbf{F} \in L^{\infty}(L(\mathcal{H}))$ we have that $\mathbf{F} \in H^{\infty}(L(\mathcal{H}))$ if and only if $M_{\mathbf{F}}\left(H^{2}(\mathcal{H})\right) \subset$ $H^{2}(\mathcal{H})$. It is an easy consequence of (3.4).

For each $\mathbf{F} \in L^{\infty}(L(\mathcal{H}))$ we define a bounded linear operator $M_{\mathbf{F}}$ on $L^{2}(\mathcal{H}):$ for $\mathbf{f} \in L^{2}(\mathcal{H})$,

$$
\left(M_{\mathbf{F}} \mathbf{f}\right)(z)=\mathbf{F}(z) \mathbf{f}(z) \quad \text { a.e. on } \mathbb{T} \text {. }
$$

In particular, for $\mathbf{f} \in L^{2}(\mathcal{H})$, we have, a.e. on $\mathbb{T}$,

$$
\left(\mathbf{M}_{z} \mathbf{f}\right)(z)=z \mathbf{f}(z) \quad \text { and } \quad\left(\mathbf{M}_{\bar{z}} \mathbf{f}\right)(z)=\bar{z} \mathbf{f}(z),
$$

that is, $\mathbf{M}_{z}=M_{z \mathbf{I}_{\mathcal{H}}}$ and $\mathbf{M}_{\bar{z}}=M_{\bar{z} \mathbf{I}_{\mathcal{H}}}$. For $\mathbf{F} \in L^{\infty}(L(\mathcal{H}))$ the adjoint function $\mathbf{F}^{*}$ is naturally defined as $\mathbf{F}^{*}(z)=\mathbf{F}(z)^{*}$ (a.e. on $\mathbb{T}$ ) and we also define $\mathbf{F}^{\#}$ by $\left(\mathbf{F}^{\#}\right)(z)=\mathbf{F}(\bar{z})^{*}$ (a.e. on $\mathbb{T}$ ). Clearly, $M_{\mathbf{F}}^{*}=M_{\mathbf{F}^{*}}$. If $\mathbf{F}: \mathbb{T} \rightarrow$ $L(\mathcal{H})$ is a constant function, $\mathbf{F}(z)=F$ a.e. on $\mathbb{T}$, we will denote by $F$ its action on $L^{2}(\mathcal{H})$.

Similarly, we define a measurable function $\mathbf{F}: \mathbb{T} \rightarrow L A(\mathcal{H})$ and denote by $L^{\infty}(L A(\mathcal{H}))$ the space of all measurable essentially bounded functions valued in the space $L A(\mathcal{H})$. For each $\mathbf{C} \in L^{\infty}(L A(\mathcal{H}))$ we define a bounded antilinear operator $A_{\mathbf{C}}$ on $L^{2}(\mathcal{H})$ : for $\mathbf{f} \in L^{2}(\mathcal{H})$,

$$
\left(A_{\mathbf{C}} \mathbf{f}\right)(z)=\mathbf{C}(z) \mathbf{f}(z) \quad \text { a.e. on } \mathbb{T} \text {. }
$$




\section{4. $\mathrm{M}_{z}$-Commuting and $\mathrm{M}_{z}$-Conjugations in $L^{2}(\mathcal{H})$}

For an arbitrary conjugation $J$ in $\mathcal{H}$ let us define two conjugations $\widetilde{\mathbf{J}}$ and $\mathbf{J}^{\star}$ on $L^{2}(\mathcal{H})$ given by

$$
(\widetilde{\mathbf{J}} \mathbf{f})(z)=J(\mathbf{f}(z)) \quad \text { a.e. on } \mathbb{T}
$$

and

$$
\left(\mathbf{J}^{\star} \mathbf{f}\right)(z)=J(\mathbf{f}(\bar{z})) \quad \text { a.e. on } \mathbb{T} \text {. }
$$

Note that for $\mathbf{f}=\sum_{n=-\infty}^{\infty} x_{n} e_{n} \in L^{2}(\mathcal{H})$

$$
\widetilde{\mathbf{J}} \mathbf{f}=\sum_{n=-\infty}^{\infty} J\left(x_{-n}\right) e_{n} \quad \text { and } \quad \mathbf{J}^{\star} \mathbf{f}=\sum_{n=-\infty}^{\infty} J\left(x_{n}\right) e_{n}
$$

Indeed, if $\widetilde{\mathbf{J}} \mathbf{f}=\sum_{n=-\infty}^{\infty} y_{n} e_{n}$ is the Fourier expansion of $\widetilde{\mathbf{J}} \mathbf{f}$, then by (3.2), for each $n \in \mathbb{Z}$ and $y \in \mathcal{H}$,

$$
\begin{aligned}
\left\langle y_{n}, y\right\rangle & =\frac{\int_{\mathbb{T}}\langle(\widetilde{\mathbf{J}} \mathbf{f})(z), y\rangle \bar{z}^{n} d m(z)=\int_{\mathbb{T}}\langle J(\mathbf{f}(z)), y\rangle \bar{z}^{n} d m(z)}{}=\overline{\int_{\mathbb{T}}\langle\mathbf{f}(z), J y\rangle z^{n} d m(z)}=\overline{\left\langle x_{-n}, J y\right\rangle}=\left\langle J\left(x_{-n}\right), y\right\rangle
\end{aligned}
$$

and so $y_{n}=J\left(x_{-n}\right)$. The Fourier coefficients of $\mathbf{J}^{\star} \mathbf{f}$ can be obtained similarly. Observe that by (4.3),

$$
\mathbf{J}^{\star}\left(H^{2}(\mathcal{H})\right) \subset H^{2}(\mathcal{H}) \text { and } \quad \widetilde{\mathbf{J}}\left(H^{2}(\mathcal{H})\right) \subset L^{2}(\mathcal{H}) \ominus z H^{2}(\mathcal{H}) .
$$

Moreover, conjugations $\widetilde{\mathbf{J}}$ and $\mathbf{J}^{\star}$ have the following properties:

Proposition 4.1. For $\mathbf{f} \in L^{2}(\mathcal{H})$ we have
1. $\left(\widetilde{\mathbf{J}} \mathbf{J}^{\star} \mathbf{f}\right)(z)=\left(\mathbf{J}^{\star} \widetilde{\mathbf{J}} \mathbf{f}\right)(z)=\mathbf{f}(\bar{z})$ for almost all $z \in \mathbb{T}$,
2. $\widetilde{\mathbf{J}} \mathbf{M}_{z}=\mathbf{M}_{\bar{z}} \widetilde{\mathbf{J}}$
3. $\mathbf{J}^{\star} \mathbf{M}_{z}=\mathbf{M}_{z} \mathbf{J}^{\star}$.

Proposition 4.2. Let $J$ be a conjugation in $\mathcal{H}$, and let $\mathbf{F} \in L^{\infty}(L(\mathcal{H}))$. Then

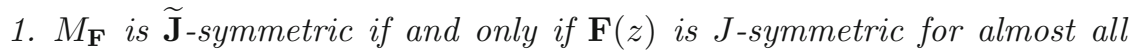
$z \in \mathbb{T}$.

2. $M_{\mathbf{F}}$ is $\mathbf{J}^{\star}$-symmetric if and only if $J \mathbf{F}(z) J=\mathbf{F}^{\#}(z)$ for almost all $z \in \mathbb{T}$.

3. $\widetilde{\mathbf{J}} M_{\mathbf{F}} \widetilde{\mathbf{J}}=M_{\mathbf{F} *}$ if and only if $\mathbf{J}^{\star} M_{\mathbf{F}} \mathbf{J}^{\star}=M_{\mathbf{F}}$.

4. $\widetilde{\mathbf{J}} M_{\mathbf{F}} \widetilde{\mathbf{J}}=M_{\mathbf{F}} \#$ if and only if $\mathbf{J}^{\star} M_{\mathbf{F}} \mathbf{J}^{\star}=M_{\mathbf{F}^{*}}$.

5. if $\mathbf{F}(\bar{z})=\mathbf{F}(z)$ for almost all $z \in \mathbb{T}$, then $M_{\mathbf{F}}$ is $\widetilde{\mathbf{J}}$-symmetric if and only if it is $\mathbf{J}^{\star}$-symmetric.

Proof. Note that for $\mathbf{f} \in L^{2}(\mathcal{H})$ we have a.e. on $\mathbb{T}$

$$
\begin{aligned}
\left(\widetilde{\mathbf{J}} M_{\mathbf{F}} \widetilde{\mathbf{J}} \mathbf{f}\right)(z) & =J\left(\left(M_{\mathbf{F}} \widetilde{\mathbf{J}} \mathbf{f}\right)(z)\right) \\
& =J(\mathbf{F}(z)(\widetilde{\mathbf{J}} \mathbf{f})(z))=J \mathbf{F}(z) J(\mathbf{f}(z)) .
\end{aligned}
$$


Hence (1) is proved. Similarly, (2) follows from the equality

$$
\begin{aligned}
\left(\mathbf{J}^{\star} M_{\mathbf{F}} \mathbf{J}^{\star} \mathbf{f}\right)(z) & =J\left(\left(M_{\mathbf{F}} \mathbf{J}^{\star} \mathbf{f}\right)(\bar{z})\right) \\
& =J\left(\mathbf{F}(\bar{z})\left(\mathbf{J}^{\star} \mathbf{f}\right)(\bar{z})\right)=J \mathbf{F}(\bar{z}) J(\mathbf{f}(z)) .
\end{aligned}
$$

Comparing (4.4) with (4.5) we get (3) and (4). Condition (5) follows from (3), since $\mathbf{F}(\bar{z})=\mathbf{F}(z)$ (a.e. on $\mathbb{T}$ ) if and only if $\mathbf{F}(z)^{*}=\mathbf{F}^{\#}(z)$ (a.e. on $\mathbb{T})$.

The following theorem gives a characterization of all $\mathbf{M}_{z \text {-commuting }}$ conjugations in $L^{2}(\mathcal{H})$.

Theorem 4.3. Let $J$ be a conjugation in $\mathcal{H}$. Then the following conditions are equivalent:

1. $\mathbf{C}$ is a conjugation in $L^{2}(\mathcal{H})$ such that $\mathbf{C M}_{z}=\mathbf{M}_{z} \mathbf{C}$,

2. there is $\mathbf{U} \in L^{\infty}(L(\mathcal{H}))$ such that $\mathbf{U}(z)$ is a unitary operator for almost all $z \in \mathbb{T}, M_{\mathbf{U}}$ is $\mathbf{J}^{\star}$-symmetric and $\mathbf{C}=M_{\mathbf{U}} \mathbf{J}^{\star}=\mathbf{J}^{\star} M_{\mathbf{U}^{*}}$.

Proof. From the equality $\mathbf{C M}_{z}=\mathbf{M}_{z} \mathbf{C}$ and Proposition 4.1 we have

$$
\mathrm{CJ}^{\star} \mathbf{M}_{z}=\mathbf{C M}_{z} \mathbf{J}^{\star}=\mathbf{M}_{z} \mathbf{C J}^{\star} .
$$

Since the linear operator $\mathbf{C J}^{\star}$ is unitary and commutes with $\mathbf{M}_{z}$, then (see [13, Theorem 3.17, Corollary 3.19]) it is equal to $M_{\mathbf{U}}$, where $\mathbf{U} \in L^{\infty}(L(\mathcal{H})$ ) and $\mathbf{U}(z)$ is unitary for almost all $z \in \mathbb{T}$. Hence $\mathbf{C}=M_{\mathbf{U}} \mathbf{J}^{\star}$. Since $\mathbf{C}$ is a conjugation, for $\mathbf{f} \in L^{2}(\mathcal{H})$ a.e. on $\mathbb{T}$ we must have

$$
\begin{aligned}
\mathbf{f}(z) & =\left(\mathbf{C}^{2} \mathbf{f}\right)(z)=\left(M_{\mathbf{U}} \mathbf{J}^{\star} M_{\mathbf{U}} \mathbf{J}^{\star} \mathbf{f}\right)(z) \\
& =\mathbf{U}(z) J\left(\left(M_{\mathbf{U}} \mathbf{J}^{\star} \mathbf{f}\right)(\bar{z})\right)=\mathbf{U}(z) J \mathbf{U}(\bar{z}) J \mathbf{f}(z),
\end{aligned}
$$

which is equivalent to $J \mathbf{U}(\bar{z}) J=\mathbf{U}(z)^{*}$ a.e. on $\mathbb{T}$. Therefore, condition $(2)$ follows from Proposition 4.2 (2).

The implication $(2) \Rightarrow(1)$ is easy.

Remark 4.4. Let us note that condition (1) in Theorem 4.3 does not depend on the conjugation $J$ in $\mathcal{H}$. Hence if $\mathbf{C}$ satisfies (1), then condition (2) is satisfied for any conjugation $J$ in $\mathcal{H}$. Therefore, for two different conjugations $J$ and $J^{\prime}$ we obtain two different unitary operator valued functions $\mathbf{U}$ and $\mathbf{U}^{\prime}$ such that

$$
\mathbf{C}=M_{\mathbf{U}} \mathbf{J}^{\star}=M_{\mathbf{U}^{\prime}} \mathbf{J}^{\prime \star} .
$$

Thus $\mathbf{U}^{\prime}(z)=\mathbf{U}(z) V_{0}$ for almost all $z \in \mathbb{T}$, where $V_{0}$ is a unitary operator given by $V_{0}=J J^{\prime}$. It follows that if we have, for a given conjugation $\mathbf{C}$, the operator valued function $\mathbf{U}$ determined by some conjugation $J$ in $\mathcal{H}$, then we can easily obtain the function $\mathbf{U}^{\prime}$ corresponding to any other conjugation $J^{\prime}$ in $\mathcal{H}$.

Recall (see [2, Theorem 2.4]) that in the scalar case $C$ commutes with $M_{z}$ if and only if $C M_{\varphi}=M_{\varphi \#} C$ for all $\varphi \in L^{\infty}$. This is not necessarily true in the general case. 
Remark 4.5. Let $\mathbf{C}$ be a conjugation in $L^{2}(\mathcal{H}), \mathbf{C}=M_{\mathbf{U}} \mathbf{J}^{\star}$ for some $\mathbf{U} \in$ $L^{\infty}(L(\mathcal{H}))$ such that $\mathbf{U}(z)$ is a unitary operator for almost all $z \in \mathbb{T}$ and $M_{\mathbf{U}}$ is $\mathbf{J}^{\star}$-symmetric. Assume that $\boldsymbol{\Phi} \in L^{\infty}(L(\mathcal{H}))$. Then

$$
\mathbf{C} M_{\Phi}=M_{\Phi *} \mathbf{C}
$$

if and only if

$$
\mathbf{U}(z) J \boldsymbol{\Phi}(\bar{z})=\boldsymbol{\Phi}^{\#}(z) \mathbf{U}(z) J
$$

for almost all $z$ in $\mathbb{T}$. Indeed, this follows from the fact that a.e. on $\mathbb{T}$,

$$
\begin{aligned}
\left(\mathbf{C} M_{\boldsymbol{\Phi}} \mathbf{f}\right)(z) & =\left(M_{\mathbf{U}} \mathbf{J}^{\star} M_{\boldsymbol{\Phi}} \mathbf{f}\right)(z)=\mathbf{U}(z)\left(\mathbf{J}^{\star} M_{\boldsymbol{\Phi}} \mathbf{f}\right)(z) \\
& =\mathbf{U}(z) J\left(\left(M_{\boldsymbol{\Phi}} \mathbf{f}\right)(\bar{z})\right)=\mathbf{U}(z) J(\boldsymbol{\Phi}(\bar{z}) \mathbf{f}(\bar{z}))
\end{aligned}
$$

and

$$
\left(M_{\mathbf{\Phi} \#} \mathbf{C f}\right)(z)=\left(M_{\mathbf{\Phi} \#} M_{\mathbf{U}} \mathbf{J}^{\star} \mathbf{f}\right)(z)=\boldsymbol{\Phi}^{\#}(z) \mathbf{U}(z) J(\mathbf{f}(\bar{z})) .
$$

If $\boldsymbol{\Phi}(z)$ is $J$-symmetric for almost all $z \in \mathbb{T}$, then $J \boldsymbol{\Phi}(\bar{z})=\boldsymbol{\Phi}^{\#}(z) J$ a.e. on $\mathbb{T}$ and condition (4.7) holds provided that for almost all $z \in \mathbb{T}, \Phi^{\#}(z)$ commutes with $\mathbf{U}(z)$.

Example 4.6. If $\mathcal{H}=\mathbb{C}$ and $J(w)=\bar{w}, w \in \mathbb{C}$, then $U \in L^{\infty}$ and, by Proposition $4.2(2), \mathbf{J}^{\star}$-symmetry of $U$ means that $U(z)=U(\bar{z})$ a.e. on $\mathbb{T}$ and we obtain $[2$, Theorem 2.4].

Example 4.7. Consider $\mathcal{H}=\mathbb{C}^{2}$ and the conjugation on $\mathbb{C}^{2}$ defined by $J_{1}\left(z_{1}, z_{2}\right)=\left(\bar{z}_{1}, \bar{z}_{2}\right)$. Then, by Theorem 2.1, any $\mathbf{M}_{z}$-commuting conjugation $\mathbf{C}$ on $L^{2}\left(\mathbb{C}^{2}\right)$ has a form

$$
\mathbf{C}=\left[\begin{array}{cc}
M_{\psi_{1}} J^{\star} & M_{\psi_{2}} J^{\star} \\
M_{\bar{\psi}_{2}^{\#}} J^{\star} & M_{\psi_{4}} J^{\star}
\end{array}\right]=\left[\begin{array}{cc}
M_{\psi_{1}} & M_{\psi_{2}} \\
M_{\bar{\psi}_{2}^{\#}} & M_{\psi_{4}}
\end{array}\right]\left[\begin{array}{cc}
J^{\star} & 0 \\
0 & J^{\star}
\end{array}\right]=M_{\mathbf{U}} \mathbf{J}_{1}^{\star}
$$

and the functions $\psi_{1}, \psi_{2}, \psi_{4} \in L^{\infty}$ satisfy conditions (2.4)-(2.6). Hence in view of Theorem 4.3 the unitary operator valued function is $\mathbf{U}=\left[\begin{array}{cc}\psi_{1} & \psi_{2} \\ \bar{\psi}_{2}^{\#} & \psi_{4}\end{array}\right]$. By conditions (2.4)-(2.6) we have that $M_{\mathbf{U}}$ is $\mathbf{J}_{1}^{\star}$-symmetric and the operator $\mathbf{U}(z)$ is unitary for almost all $z \in \mathbb{T}$.

On the other hand, if the conjugation on $\mathbb{C}^{2}$ is defined by $J_{2}\left(z_{1}, z_{2}\right)=$ $\left(\bar{z}_{2}, \bar{z}_{1}\right)$, then $\mathbf{J}_{2}^{\star}=\left[\begin{array}{cc}0 & J^{\star} \\ J^{\star} & 0\end{array}\right]$. The conjugation $\mathbf{C}$ on $L^{2}\left(\mathbb{C}^{2}\right)$ has the form

$$
\begin{aligned}
\mathbf{C}=\left[\begin{array}{ll}
M_{\psi_{1}} & M_{\psi_{2}} \\
M_{\bar{\psi}_{2}^{\#}} & M_{\psi_{4}}
\end{array}\right]\left[\begin{array}{cc}
J^{\star} & 0 \\
0 & J^{\star}
\end{array}\right] & =\left[\begin{array}{ll}
M_{\psi_{1}} & M_{\psi_{2}} \\
M_{\bar{\psi}_{2}^{\#}} & M_{\psi_{4}}
\end{array}\right]\left[\begin{array}{ll}
0 & 1 \\
1 & 0
\end{array}\right]\left[\begin{array}{cc}
0 & J^{\star} \\
J^{\star} & 0
\end{array}\right] \\
& =\left[\begin{array}{ll}
M_{\psi_{2}} & M_{\psi_{1}} \\
M_{\psi_{4}} & M_{\bar{\psi}_{2}^{\#}}^{\#}
\end{array}\right] \mathbf{J}_{2}^{\star}=M_{\mathbf{U}_{2}} \mathbf{J}_{2}^{\star} .
\end{aligned}
$$

By straightforward calculations one can check that $M_{\mathbf{U}_{2}}$ is $\mathbf{J}_{2}^{\star}$-symmetric and $\mathbf{U}_{2}(z)$ is unitary for almost all $z \in \mathbb{T}$.

The following theorem gives a characterization of all $\mathbf{M}_{z}$-conjugations in $L^{2}(\mathcal{H})$. 
Theorem 4.8. Let $\mathbf{C}$ be an antilinear operator in $L^{2}(\mathcal{H})$. Then the following are equivalent

1. $\mathbf{C}$ is a conjugation on $L^{2}(\mathcal{H})$ such that $\mathbf{M}_{z} \mathbf{C}=\mathbf{C} \mathbf{M}_{\bar{z}}$,

2. there is $\mathbf{C}_{0} \in L^{\infty}(L A(\mathcal{H}))$ such that $\mathbf{C}=A_{\mathbf{C}_{0}}$ and $\mathbf{C}_{0}(z)$ is a conjugation for almost all $z \in \mathbb{T}$,

3. for any conjugation $J$ in $\mathcal{H}$ there is $\mathbf{U} \in L^{\infty}(L(\mathcal{H}))$ such that $\mathbf{U}(z)$ is a J-symmetric unitary operator for almost all $z \in \mathbb{T}$ and $\mathbf{C}=M_{\mathbf{U}} \widetilde{\mathbf{J}}=$ $\widetilde{\mathbf{J}} M_{\mathbf{U}^{*}}$.

Proof. First we will prove that $(1) \Rightarrow(3)$. By Proposition 4.1 (2) we have

$$
\mathbf{M}_{z} \mathbf{C} \widetilde{\mathbf{J}}=\mathbf{C} \mathbf{M}_{\bar{z}} \widetilde{\mathbf{J}}=\mathbf{C} \widetilde{\mathbf{J}} \mathbf{M}_{z}
$$

which implies that the unitary operator $\mathbf{C} \widetilde{\mathbf{J}}$ commutes with $\mathbf{M}_{z}$. Hence, as in the proof of Theorem 4.3, $\mathbf{C} \widetilde{\mathbf{J}}=M_{\mathbf{U}}$, for $\mathbf{U} \in L^{\infty}(L(\mathcal{H}))$ such that $\mathbf{U}(z)$ is unitary for almost all $z \in \mathbb{T}$. Then $\mathbf{C}=M_{\mathbf{U}} \widetilde{\mathbf{J}}$. Since $\mathbf{C}$ is a conjugation, for almost all $z \in \mathbb{T}$ we must have

$$
\begin{aligned}
\mathbf{f}(z) & =\left(\mathbf{C}^{2} \mathbf{f}\right)(z)=\left(M_{\mathbf{U}} \widetilde{\mathbf{J}} M_{\mathbf{U}} \widetilde{\mathbf{J}} \mathbf{f}\right)(z) \\
& =\mathbf{U}(z) J\left(\left(M_{\mathbf{U}} \widetilde{\mathbf{J}} \mathbf{f}\right)(z)\right)=\mathbf{U}(z) J \mathbf{U}(z) J \mathbf{f}(z),
\end{aligned}
$$

which is equivalent to $J \mathbf{U}(z) J=\mathbf{U}(z)^{*}$ a.e. on $\mathbb{T}$.

To see that $(3) \Rightarrow(2)$ define $\mathbf{C}_{0} \in L^{\infty}(L A(\mathcal{H}))$ as $\mathbf{C}_{0}(z)=\mathbf{U}(z) J$ a.e. on $\mathbb{T}$. An easy calculation shows that $(2) \Rightarrow(1)$.

Remark 4.9. As in Theorem 4.3, condition (1) in Theorem 4.8 does not depend on $J$. Hence (1) implies that for any conjugations $J$ and $J^{\prime}$ in $\mathcal{H}$ there exist unitary operator valued functions $\mathbf{U}$ and $\mathbf{U}^{\prime}$ such that

$$
\mathbf{C}=M_{\mathbf{U}} \widetilde{\mathbf{J}}=M_{\mathbf{U}^{\prime}} \widetilde{\mathbf{J}}^{\prime}
$$

Moreover, $\mathbf{U}^{\prime}(z)=\mathbf{U}(z) J J^{\prime}$ a.e. on $\mathbb{T}$.

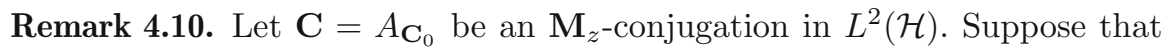
$\mathbf{F} \in L^{\infty}(L(\mathcal{H}))$ is an operator valued function. Then $M_{\mathbf{F}}$ is $\mathbf{C}$-symmetric if and only if for almost all $z \in \mathbb{T}, \mathbf{F}(z)$ is $\mathbf{C}_{0}(z)$-symmetric.

Example 4.11. Consider $\mathcal{H}=\mathbb{C}^{2}$ and the conjugation $J_{1}$ in $\mathbb{C}^{2}$ defined by

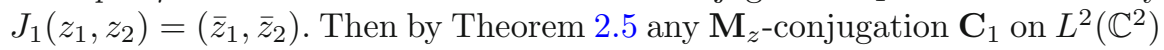
has a form

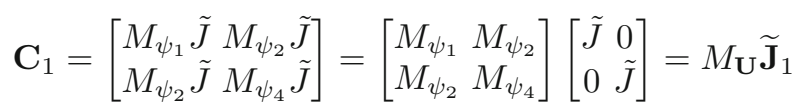

and the functions $\psi_{1}, \psi_{2}, \psi_{4} \in L^{\infty}$ satisfy conditions (2.9)-(2.10). Hence in view of Theorem 4.8 , the unitary operator valued function is

$$
\mathbf{U}=\left[\begin{array}{ll}
\psi_{1} & \psi_{2} \\
\psi_{2} & \psi_{4}
\end{array}\right]
$$

If we consider another conjugation in $\mathbb{C}^{2}$ given by $J_{2}\left(z_{1}, z_{2}\right)=\left(\bar{z}_{2}, \bar{z}_{1}\right)$, then the conjugation $\mathbf{C}_{2}$ in $L^{2}\left(\mathbb{C}^{2}\right)$ has a form

$$
\mathbf{C}_{2}=\left[\begin{array}{ll}
M_{\psi_{2}} & M_{\psi_{1}} \\
M_{\psi_{4}} & M_{\psi_{2}}
\end{array}\right]\left[\begin{array}{cc}
0 & \tilde{J} \\
\tilde{J} & 0
\end{array}\right]=M_{\mathbf{U}_{2}} \widetilde{\mathbf{J}}_{2} \text {. }
$$




\section{Conjugations Preserving $H^{2}(\mathcal{H})$}

Let $J$ be any conjugation in $\mathcal{H}$. Then, as noted before, by (4.3) we have $\mathbf{J}^{\star}\left(H^{2}(\mathcal{H})\right) \subset H^{2}(\mathcal{H})$. Now our aim is to characterize all $\mathbf{M}_{z}$-commuting conjugations with this property. Observe here that if $\mathbf{C}$ is a conjugation in $L^{2}(\mathcal{H})$ such that $\mathbf{C}\left(H^{2}(\mathcal{H})\right) \subset H^{2}(\mathcal{H})$, then actually we must have $\mathbf{C}\left(H^{2}(\mathcal{H})\right)=H^{2}(\mathcal{H})$.

Theorem 5.1. Let $J$ be a conjugation in $\mathcal{H}$ and let $\mathbf{C}$ be a conjugation in $L^{2}(\mathcal{H})$ such that $\mathbf{C M}_{z}=\mathbf{M}_{z} \mathbf{C}$. If $\mathbf{C}\left(H^{2}(\mathcal{H})\right) \subset H^{2}(\mathcal{H})$, then there is a unitary $J$-symmetric operator $U_{0} \in L(\mathcal{H})$ such that $\mathbf{C}=M_{\mathbf{U}^{\star}} \mathbf{J}^{\star}=\mathbf{J}^{\star} M_{\mathbf{U}^{*}}$, where $\mathbf{U}$ is a constant operator valued function $\mathbf{U}(z)=U_{0}$ for almost all $z \in \mathbb{T}$.

Proof. By Theorem 4.3, there exists a unitary valued $\mathbf{U} \in L^{\infty}(L(\mathcal{H}))$ such that $\mathbf{C}=M_{\mathbf{U}} \mathbf{J}^{\star}$ and $M_{\mathbf{U}} \mathbf{M}_{z}=\mathbf{M}_{z} M_{\mathbf{U}}$. Since, by assumption, $M_{\mathbf{U}} \mathbf{J}^{\star}\left(H^{2}(\mathcal{H})\right) \subset H^{2}(\mathcal{H})$, we have $M_{\mathbf{U}} H^{2}(\mathcal{H}) \subset H^{2}(\mathcal{H})$. By the commutativity relation

$$
M_{\mathbf{U}} \mathbf{M}_{z^{k}}=\mathbf{M}_{z^{k}} M_{\mathbf{U}}
$$

we get that

$$
M_{\mathbf{U}}\left(z^{k} H^{2}(\mathcal{H})\right) \subset z^{k} H^{2}(\mathcal{H})
$$

for every nonnegative integer $k$. Since $\mathbf{C}=\mathbf{C}^{\sharp}=\mathbf{J}^{\star} M_{\mathbf{U}^{*}}$, similarly,

$$
M_{\mathbf{U}^{*}}\left(z^{k} H^{2}(\mathcal{H})\right) \subset z^{k} H^{2}(\mathcal{H})
$$

which implies that all subspaces $z^{k} H^{2}(\mathcal{H}), k=0,1, \ldots$, are reducing for $M_{\mathbf{U}}$. Hence also $H^{2}(\mathcal{H}) \ominus z H^{2}(\mathcal{H})$ is reducing for $M_{\mathbf{U}}$. Therefore

$$
M_{\mathbf{U}} \mathcal{H} \subset \mathcal{H}
$$

which means that $\mathbf{U}$ is a constant operator valued function, $\mathbf{U}(z)=U_{0}$ for almost all $z \in \mathbb{T}$, where $U_{0} \in L(\mathcal{H})$ is a unitary operator. Since $M_{\mathbf{U}}$ is $\mathbf{J}^{\star}$ symmetric, we get that $U_{0}$ is $J$-symmetric.

Remark 5.2. If $\mathcal{H}=\mathbb{C}$ and $J(w)=\bar{w}, w \in \mathbb{C}$, then $U_{0}$ is a constant of modulus 1 (see [2, Corollary 3.1]).

Example 5.3. As in Example 4.7 let $\mathcal{H}=\mathbb{C}^{2}$ and $J_{1}\left(z_{1}, z_{2}\right)=\left(\bar{z}_{1}, \bar{z}_{2}\right)$. Then, by Theorem 5.1, Example 4.7 and conditions (2.4)-(2.6), an antilinear operator $\mathbf{C}=\left[\begin{array}{ll}D_{1} & D_{2} \\ D_{2}^{\#} & D_{4}\end{array}\right], i=1,2,4$, is a conjugation commuting with $\mathbf{M}_{z}$ and $\mathbf{C}\left(H^{2}\left(\mathbb{C}^{2}\right)\right) \subset H^{2}\left(\mathbb{C}^{2}\right)$ if and only if $D_{i}=\lambda_{i} J^{\star}$ with $\lambda_{i} \in \mathbb{D}$ such that

$$
\begin{aligned}
& \left|\lambda_{1}\right|^{2}+\left|\lambda_{2}\right|^{2}=\left|\lambda_{2}\right|^{2}+\left|\lambda_{4}\right|^{2}=1 \\
& \bar{\lambda}_{1} \lambda_{2}+\bar{\lambda}_{2} \lambda_{4}=0 .
\end{aligned}
$$

Remark 5.4. Note that if $\mathbf{C}$ is an $\mathbf{M}_{z}$-commuting conjugation such that $\mathbf{C}\left(H^{2}(\mathcal{H})\right) \subset H^{2}(\mathcal{H})$, then for every conjugation $J$ in $\mathcal{H}$ there is a unitary $J$-symmetric operator $U_{0} \in L(\mathcal{H})$ such that $\mathbf{C}=\mathbf{M}_{\mathbf{U}} \mathbf{J}^{\star}$, where $\mathbf{U}$ is a constant operator valued function $\mathbf{U}(z)=U_{0}$ for almost all $z \in \mathbb{T}$. In view of Remark 4.4 the relation between the values of constant operator valued functions corresponding to conjugations $J_{1}$ and $J_{2}$ is given by $U_{2}=U_{1} J_{1} J_{2}$. 
Considering $\mathbf{M}_{z}$-conjugations preserving $H^{2}(\mathcal{H})$ note that $\widetilde{\mathbf{J}}\left(H^{2}(\mathcal{H})\right) \not \subset$ $H^{2}(\mathcal{H})$. In fact, $\widetilde{\mathbf{J}}\left(H^{2}(\mathcal{H})\right)=L^{2}(\mathcal{H}) \ominus z H^{2}(\mathcal{H})$. More generally, we have:

Proposition 5.5. There are no $\mathbf{M}_{z}$-conjugations on $L^{2}(\mathcal{H})$ for which $H^{2}(\mathcal{H})$ is invariant.

Proof. Assume that $\mathbf{C}$ is an $\mathbf{M}_{z}$-conjugation and $\mathbf{C}\left(H^{2}(\mathcal{H})\right) \subset H^{2}(\mathcal{H})$. Then $\mathbf{C}\left(H^{2}(\mathcal{H})\right)=H^{2}(\mathcal{H})$. By Theorem $4.8(3), \mathbf{C}=M_{\mathbf{U}} \widetilde{\mathbf{J}}$, hence

$$
M_{\mathbf{U}}\left(H^{2}(\mathcal{H})\right)=\mathbf{C} \widetilde{\mathbf{J}}\left(H^{2}(\mathcal{H})\right)=L^{2}(\mathcal{H}) \ominus z H^{2}(\mathcal{H}) .
$$

Let $\mathbf{f} \in H^{2}(\mathcal{H})$. Then also $z^{k} \mathbf{f} \in H^{2}(\mathcal{H})$ for any $k=1,2, \ldots$ Since $\mathbf{U}$ is an operator valued function, $M_{\mathbf{U}}$ commutes with $\mathbf{M}_{z}$ and $\mathbf{M}_{\bar{z}}$. Hence $M_{\mathbf{U}}\left(z^{k} \mathbf{f}\right)=z^{k} M_{\mathbf{U}} \mathbf{f} \perp z H^{2}(\mathcal{H})$ and $M_{\mathbf{U}} \mathbf{f} \perp \bar{z}^{k-1} H^{2}(\mathcal{H})$. Since $k$ is arbitrary, then $M_{\mathbf{U}} \mathbf{f}=0$, so we also have $M_{\mathbf{U}}=0$, which is a contradiction.

\section{Conjugations and Model Spaces}

Assume now that $\operatorname{dim} \mathcal{H}=d<\infty$. A function $\Theta \in H^{\infty}(L(\mathcal{H}))$ is called inner, if its boundary values are unitary operators in $L(\mathcal{H})$ almost everywhere on $\mathbb{T}$ (since $\operatorname{dim} \mathcal{H}<\infty)$. Suppose that $\Theta$ is a pure inner function, i.e., $\|\Theta(0)\|<1$. Define the corresponding model space $K_{\Theta}=H^{2}(\mathcal{H}) \ominus \Theta H^{2}(\mathcal{H})$ and let $P_{\Theta}$ be the orthogonal projection on $K_{\Theta}$. Note that the subspace $K_{\Theta}^{\infty}$ of all bounded functions in $K_{\Theta}\left(K_{\Theta}^{\infty}=K_{\Theta} \cap L^{\infty}(\mathcal{H})\right)$ is dense in $K_{\Theta}$.

Lemma 6.1. Let $\mathbf{F} \in L^{\infty}(L(\mathcal{H}))$ and let $J_{1}, J_{2}$ be any two conjugations in $\mathcal{H}$. Then $\widetilde{\mathbf{F}}$ defined a.e. on $\mathbb{T}$ by $\widetilde{\mathbf{F}}(z)=J_{1} \mathbf{F}(z) J_{2}$ belongs to $H^{\infty}(L(\mathcal{H}))$ if and only if $\mathbf{F}^{*}$ belongs to $H^{\infty}(L(\mathcal{H}))$.

Proof. It is clear that $\widetilde{\mathbf{F}} \in L^{\infty}(L(\mathcal{H}))$ whenever $\mathbf{F} \in L^{\infty}(L(\mathcal{H}))$. Let $\mathbf{F} \in$ $L^{\infty}(L(\mathcal{H}))$ be given by expansion (3.3). Then, by (3.4), the $n$-th Fourier coefficient of $\widetilde{\mathbf{F}}$ equals

$$
(\widetilde{\mathbf{F}})_{n} h=\int_{\mathbb{T}} z^{-n} J_{1} \mathbf{F}(z) J_{2} h d m(z)=J_{1} \int_{\mathbb{T}} z^{n} \mathbf{F}(z) J_{2} h d m(z)=J_{1} F_{-n} J_{2} h
$$

for $h \in \mathcal{H}$. On the other hand, also by (3.4), $n$-th Fourier coefficient of $\mathbf{F}^{*}$ equals $\left(\mathbf{F}^{*}\right)_{n}=F_{-n}^{*}$. Now the rest of the proof is clear.

Lemma 6.2. (see [1], pp. 118-119) Let $\Theta \in H^{\infty}(L(\mathcal{H}))$ be an operator valued inner function and let $\mathbf{F} \in L^{\infty}(L(\mathcal{H}))$. If $M_{\mathbf{F}}\left(H^{2}(\mathcal{H})\right) \subset \Theta H^{2}(\mathcal{H})$, then $\mathbf{F} \in H^{\infty}(L(\mathcal{H}))$ and there exists $\Psi \in H^{\infty}(L(\mathcal{H}))$ such that $\mathbf{F}=\Theta \Psi$.

Proof. The proof follows the reasoning presented in [1, pp. 118-119]. The inclusion $M_{\mathbf{F}}\left(H^{2}(\mathcal{H})\right) \subset \Theta H^{2}(\mathcal{H})$ means that for each $\mathbf{f} \in H^{2}(\mathcal{H})$ there exists $\mathbf{g} \in H^{2}(\mathcal{H})$ such that

$$
M_{\mathbf{F}} \mathbf{f}=M_{\Theta} \mathbf{g} .
$$

Moreover, since $M_{\Theta}$ is an isometry, there is only one such $\mathbf{g}$ and

$$
\|\mathbf{g}\|=\left\|M_{\Theta} \mathbf{g}\right\|=\left\|M_{\mathbf{F}} \mathbf{f}\right\| \leqslant\|\mathbf{F}\|_{\infty}\|\mathbf{f}\| .
$$


We can thus define a bounded linear operator $T: H^{2}(\mathcal{H}) \rightarrow H^{2}(\mathcal{H})$ by $\mathbf{f} \mapsto$ $T \mathbf{f}=\mathbf{g}$. Hence, for $\mathbf{f} \in H^{2}(\mathcal{H})$ we have $M_{\mathbf{F}} \mathbf{f}=M_{\Theta} T \mathbf{f}$ and

$$
M_{\Theta} T \mathbf{M}_{z} \mathbf{f}=M_{\mathbf{F}} \mathbf{M}_{z} \mathbf{f}=\mathbf{M}_{z} M_{\mathbf{F}} \mathbf{f}=\mathbf{M}_{z} M_{\Theta} T \mathbf{f}=M_{\Theta} \mathbf{M}_{z} T \mathbf{f}
$$

which means that $T \mathbf{M}_{z} \mathbf{f}=\mathbf{M}_{z} T \mathbf{f}$. It follows that $T=M_{\Psi}$ for some $\Psi \in$ $H^{\infty}(L(\mathcal{H}))\left(\left[1\right.\right.$, Chap. 5, Theorem 1.7]) and $M_{\mathbf{F}} \mathbf{f}=M_{\Theta \Psi} \mathbf{f}$. In particular, for every $x \in \mathcal{H}$,

$$
\mathbf{F}(z) x=\Theta(z) \Psi(z) x \quad \text { a.e. on } \mathbb{T}
$$

and so $\mathbf{F} \in H^{\infty}(L(\mathcal{H}))$.

We can now give another proof of Lemma 6.1. Namely, if $\widetilde{\mathbf{F}} \in$ $H^{\infty}(L(\mathcal{H}))$, then $M_{\widetilde{\mathbf{F}}}\left(z H^{2}(\mathcal{H})\right) \subset z H^{2}(\mathcal{H})$. Since $\mathbf{F}(z)=J_{1} \widetilde{\mathbf{F}}(z) J_{2}$ a.e. on $\mathbb{T}$, we have that $M_{\mathbf{F}}=\widetilde{\mathbf{J}}_{1} M_{\widetilde{\mathbf{F}}} \widetilde{\mathbf{J}}_{2}$ and

$$
\begin{aligned}
M_{\mathbf{F}}\left(H^{2}(\mathcal{H})^{\perp}\right) & =\widetilde{\mathbf{J}}_{1} M_{\widetilde{\mathbf{F}}} \widetilde{\mathbf{J}}_{2}\left(H^{2}(\mathcal{H})^{\perp}\right)=\widetilde{\mathbf{J}}_{1} M_{\widetilde{\mathbf{F}}}\left(z H^{2}(\mathcal{H})\right) \\
& \subset \widetilde{\mathbf{J}}_{1}\left(z H^{2}(\mathcal{H})\right)=H^{2}(\mathcal{H})^{\perp}
\end{aligned}
$$

It follows that $M_{\mathbf{F}^{*}}=M_{\mathbf{F}}^{*}$ preserves $H^{2}(\mathcal{H})$ and $\mathbf{F}^{*} \in H^{\infty}(L(\mathcal{H}))$ by Lemma 6.2. The proof of the other implication is analogous.

Lemma 6.3. Let $\mathbf{F} \in H^{\infty}(L(\mathcal{H}))$ and let $J$ be a conjugation in $\mathcal{H}$. Then the following are equivalent:

1. $\mathbf{F}(z)$ is J-symmetric a.e. on $\mathbb{T}$;

2. $\mathbf{F}(\lambda)$ is J-symmetric for all $\lambda \in \mathbb{D}$.

Proof. For the proof it is enough to note that each of the conditions (1) and $(2)$ is equivalent to $J$-symmetry of all of the coefficients $F_{n}$ of the Fourier/Taylor expansion of $\mathbf{F}$.

In what follows, if the operator valued inner function $\Theta$ and the conjugation $J$ satisfy one of the conditions (1) or (2) from Lemma 6.3 we will simply say that $\Theta$ is $J$-symmetric.

The model operator $S_{\Theta} \in L\left(K_{\Theta}\right)$ is given by

$$
\left(S_{\Theta} \mathbf{f}\right)=P_{\Theta}\left(\mathbf{M}_{z} \mathbf{f}\right) \quad \text { for } \mathbf{f} \in K_{\Theta} .
$$

From [4, Theorem 3.1] it follows that the model operator $S_{\Theta}$ is complex symmetric if and only if there is a conjugation $J$ in $\mathcal{H}$ such that $\Theta$ is $J$ symmetric (which implies that $\Theta(z)$ is $J$-symmetric a.e. on $\mathbb{T}$ ). In that case $\Theta(z) J$ is a conjugation in $\mathcal{H}$ for almost all $z \in \mathbb{T}$ and as a consequence $M_{\Theta} \widetilde{\mathbf{J}}$ is a conjugation in $L^{2}(\mathcal{H})$ by Theorem 4.8. From now on let us assume that $\Theta$ is $J$-symmetric. Then $\mathbf{C}_{\Theta, J}$ defined by

$$
\mathbf{C}_{\Theta, J} \mathbf{f}(z)=\Theta(z) \bar{z}(\widetilde{\mathbf{J}} \mathbf{f})(z)=\Theta(z) \bar{z} J(\mathbf{f}(z)) \quad \text { a.e. on } \mathbb{T}
$$

(that is, $\mathbf{C}_{\Theta, J}=M_{\Theta} \mathbf{M}_{\bar{z}} \widetilde{\mathbf{J}}$ ) is a conjugation on $L^{2}(\mathcal{H})$ that leaves $K_{\Theta}$ invariant. Moreover, the following is true: 
Proposition 6.4. Let $\mathbf{V} \in L^{\infty}(L(\mathcal{H}))$ be a unitary operator valued function. Then $\mathbf{C}=M_{\mathbf{V}} \mathbf{C}_{\Theta, J}$ is a conjugation in $L^{2}(\mathcal{H})$ if and only if $M_{\mathbf{V}}$ is $\mathbf{C}_{\Theta, J^{-}}$ symmetric. In other words,

$$
\Theta(z) J \mathbf{V}(z) \Theta(z) J=\mathbf{V}(z)^{*}
$$

almost everywhere on $\mathbb{T}$. Moreover, if for almost all $z \in \mathbb{T}, \mathbf{V}(z)=V_{0}$, where $V_{0}$ is a unitary operator in $L(\mathcal{H})$, then $K_{\Theta}$ is invariant for $\mathbf{C}$.

Proof. Note that (6.2) is a consequence of $\mathbf{C}$ being an involution. For the proof of the second statement note that by (6.2) and $J$-symmetry of $\Theta$,

$$
V_{0} \Theta(z)=\Theta(z) J V_{0}^{*} J \quad \text { a.e. on } \mathbb{T} \text {. }
$$

So for $n \geqslant 1$ and $x \in \mathcal{H}$,

$$
\begin{aligned}
M_{\mathbf{V}} \mathbf{C}_{\Theta, J}\left(x e_{-n}\right) & =\mathbf{C}_{\Theta, J} M_{\mathbf{V}^{*}}\left(x e_{-n}\right)=\mathbf{C}_{\Theta, J}\left(V_{0}^{*} x e_{-n}\right) \\
& =M_{\Theta} \mathbf{M}_{\bar{z}} \widetilde{\mathbf{J}}\left(V_{0}^{*} x e_{-n}\right)=M_{\Theta}\left(J V_{0}^{*} x e_{n-1}\right) .
\end{aligned}
$$

Hence $\mathbf{C}\left(L^{2}(\mathcal{H}) \ominus H^{2}(\mathcal{H})\right) \subset \Theta H^{2}(\mathcal{H})$. On the other hand, for $n \geqslant 0$ and $x \in \mathcal{H}$,

$$
\begin{aligned}
M_{\mathbf{V}} \mathbf{C}_{\Theta, J} M_{\Theta}\left(x e_{n}\right) & =M_{\mathbf{V}} M_{\Theta *} \mathbf{C}_{\Theta, J}\left(x e_{n}\right)=M_{\mathbf{V}} M_{\Theta *} M_{\Theta} \mathbf{M}_{\bar{z}} \widetilde{\mathbf{J}}\left(x e_{n}\right) \\
& =M_{\mathbf{V}}\left(J x e_{-n-1}\right)=V_{0} J x e_{-n-1} .
\end{aligned}
$$

Hence $\mathbf{C}\left(\Theta H^{2}(\mathcal{H})\right) \subset L^{2}(\mathcal{H}) \ominus H^{2}(\mathcal{H})$.

For $\lambda \in \mathbb{D}$ define an operator valued function $k_{\lambda}^{\Theta}$ by

$$
k_{\lambda}^{\Theta}(z)=\frac{1}{1-\bar{\lambda} z}\left(1-\Theta(z) \Theta(\lambda)^{*}\right) \text { a.e. on } \mathbb{T} \text {. }
$$

For each $x \in \mathcal{H}$ denote the function $z \mapsto k_{\lambda}^{\Theta}(z) x$ by $k_{\lambda}^{\Theta} x$. Recall from [10] that $k_{\lambda}^{\Theta} x \in K_{\Theta}$ and for each $f \in K_{\Theta}$,

$$
\left\langle f, k_{\lambda}^{\Theta} x\right\rangle=\langle f(\lambda), x\rangle
$$

(the inner product on the left hand side is the $L^{2}(\mathcal{H})$ inner product while the inner product on the right hand side is the inner product from $\mathcal{H}$ ). Similarly, denote by $\widetilde{k_{\lambda}^{\Theta}} x$ the function $z \mapsto \widetilde{k_{\lambda}^{\Theta}}(z) x$, where

$$
\widetilde{k_{\lambda}^{\Theta}}(z)=\frac{1}{z-\lambda}(\Theta(z)-\Theta(\lambda)) \quad \text { a.e. on } \mathbb{T} \text {. }
$$

For $\Theta \in H^{\infty}(L(\mathcal{H}))$ recall that $\Theta^{\#}(z)=\Theta(\bar{z})^{*}$, and $\Theta$ is inner if and only if $\Theta^{\#}$ is inner.

Lemma 6.5. Let $J$ be a conjugation on $\mathcal{H}$ such that $\Theta$ is $J$-symmetric. Then $\mathbf{C}_{\Theta, J}\left(k_{\lambda}^{\Theta} x\right)=\widetilde{k_{\lambda}^{\Theta}} J x$ for $x \in \mathcal{H}$.

Proof. Note that a.e. on $\mathbb{T}$,

$$
\begin{aligned}
\left(\mathbf{C}_{\Theta, J} k_{\lambda}^{\Theta} x\right)(z) & =\Theta(z) \bar{z} J\left(k_{\lambda}^{\Theta}(z) x\right)=\Theta(z) \bar{z} J\left(\frac{1}{1-\bar{\lambda} z}\left(1-\Theta(z) \Theta(\lambda)^{*}\right) x\right) \\
& =\frac{\bar{z}}{1-\lambda \bar{z}} \Theta(z)\left(1-\Theta(z)^{*} \Theta(\lambda)\right) J x=\frac{1}{z-\lambda}(\Theta(z)-\Theta(\lambda)) J x
\end{aligned}
$$


Proposition 6.4 describes a class of conjugations in $L^{2}(\mathcal{H})$ which leave model spaces invariant. The following result says that amongst all $\mathbf{M}_{z^{-}}$ conjugations only conjugations in that class have this property.

Theorem 6.6. Let $\mathbf{C}$ be an $\mathbf{M}_{z}$-conjugation on $L^{2}(\mathcal{H})$ and let $\Theta \in L^{\infty}(L(\mathcal{H}))$ be a J-symmetric pure inner function with a conjugation $J$ on $\mathcal{H}$. Suppose that $K_{\Theta}$ is invariant for $\mathbf{C}$. Then $\mathbf{C}=M_{\mathbf{V}} \mathbf{C}_{\Theta, J}$ with $\mathbf{V}$ a unitary valued constant function, $\mathbf{V}(z)=V_{0}$ a.e. on $\mathbb{T}$, such that $M_{\mathbf{V}}$ is $\mathbf{C}_{\Theta, J}$-symmetric.

Proof. By Theorem 4.8 we know that $\mathbf{C}=M_{\mathbf{U}} \widetilde{\mathbf{J}}=\widetilde{\mathbf{J}} M_{\mathbf{U}^{*}}$, where $\mathbf{U} \in$ $L^{\infty}(L(\mathcal{H}))$, and for almost all $z \in \mathbb{T}, \mathbf{U}(z)$ is unitary and $J$-symmetric. Fix $x \in \mathcal{H}$. Note that a.e. on $\mathbb{T}$,

$$
\begin{aligned}
\left(\mathbf{C C}_{\Theta, J} k_{\lambda}^{\Theta} x\right)(z) & =\mathbf{U}(z) J\left(\frac{1}{z-\lambda}(\Theta(z)-\Theta(\lambda))\right) J x \\
& =\mathbf{U}(z) \frac{1}{\bar{z}-\bar{\lambda}}\left(\Theta(z)^{*}-\Theta(\lambda)^{*}\right) x \\
& =\frac{z}{1-\lambda z} \mathbf{U}(z) \Theta(z)^{*}\left(1-\Theta(z) \Theta(\lambda)^{*}\right) x \\
& =z \mathbf{U}(z) \Theta(z)^{*} k_{\lambda}^{\Theta}(z) x .
\end{aligned}
$$

Denote by $\mathbf{W}_{1}$ the operator valued function given by

$$
\mathbf{W}_{1}(z)=z \mathbf{U}(z) \Theta(z)^{*}\left(1-\Theta(z) \Theta(0)^{*}\right) \text { a.e. on } \mathbb{T} \text {. }
$$

Since $\mathbf{C C}_{\Theta, J} k_{0}^{\Theta} x \in K_{\Theta}$, then $M_{\mathbf{W}_{1}} x \in H^{2}(\mathcal{H})$. By commutativity of $M_{\mathbf{W}_{1}}$ with $\mathbf{M}_{z}$, we get that for $n=0,1, \ldots$,

$$
M_{\mathbf{W}_{1}}\left(e_{n} x\right) \in H^{2}(\mathcal{H}) .
$$

Since $x \in \mathcal{H}$ is arbitrary, hence $H^{2}(\mathcal{H})$ is invariant for $M_{\mathbf{W}_{1}}$.

Recalling that $\Theta$ is a pure analytic function, i.e., $\|\Theta(0)\|<1$, we have that $z \mapsto\left(1-\Theta(z) \Theta(0)^{*}\right)^{-1}$ is a bounded analytic function. Hence for any $\mathbf{f} \in H^{2}(\mathcal{H})$ we have $\left(1-\Theta(\cdot) \Theta(0)^{*}\right)^{-1} \mathbf{f} \in H^{2}(\mathcal{H})$ and

$$
M_{\mathbf{W}_{1}}\left(1-\Theta(\cdot) \Theta(0)^{*}\right)^{-1} \mathbf{f}=z M_{\mathbf{U}} \Theta(\cdot)^{*} \mathbf{f} \in H^{2}(\mathcal{H}) .
$$

Therefore, $\mathbf{V}=\mathbf{M}_{z} \mathbf{U} \Theta^{*}$ is analytic. On the other hand, a.e. on $\mathbb{T}$,

$$
\begin{aligned}
\left(\mathbf{C}_{\Theta, J} \mathbf{C} k_{0}^{\Theta} x\right)(z) & =\left(\mathbf{C}_{\Theta, J} \widetilde{\mathbf{J}} M_{\mathbf{U}^{*}} k_{0}^{\Theta} x\right)(z) \\
& =\Theta(z) \bar{z} \mathbf{U}(z)^{*}\left(1-\Theta(z) \Theta(0)^{*}\right) x \\
& =\Theta(z) \mathbf{U}(z)^{*} \bar{z}\left(1-\Theta(z) \Theta(0)^{*}\right) x .
\end{aligned}
$$

Similarly to what was done above we define $\mathbf{W}_{2}(z)=\Theta(z) \mathbf{U}(z)^{*} \bar{z}(1-$ $\left.\Theta(z) \Theta(0)^{*}\right)$ a.e. on $\mathbb{T}$. Observe that $M_{\mathbf{W}_{2}} \mathbf{M}_{z}=\mathbf{M}_{z} M_{\mathbf{W}_{2}}$, and consequently as above $M_{\mathbf{W}_{2}}\left(H^{2}(\mathcal{H})\right) \subset H^{2}(\mathcal{H})$. Hence for $\mathbf{f} \in H^{2}(\mathcal{H})$ we get

$$
M_{\mathbf{W}_{2}}\left(1-\Theta(\cdot) \Theta(0)^{*}\right)^{-1} \mathbf{f}=M_{\Theta} M_{\mathbf{U} *} \mathbf{M}_{\bar{z}} \mathbf{f} \in H^{2}(\mathcal{H}) .
$$

Therefore, $\mathbf{V}^{*}=\Theta \mathbf{U}^{*} \mathbf{M}_{\bar{z}}$ is also analytic. It follows that $\mathbf{V}$ is a constant unitary operator valued function, $\mathbf{V}(z)=V_{0}$ a.e. on $\mathbb{T}$. A direct calculation shows that $V_{0}$ satisfies (6.2). Hence $\mathbf{C}=M_{\mathbf{V}} \mathbf{C}_{\Theta, J}$.

Remark 6.7. Let $\Theta \in L^{\infty}(L(\mathcal{H}))$ be a pure inner function and let $\mathbf{C}$ be an $\mathbf{M}_{z}$-conjugation in $L^{2}(\mathcal{H})$ which leaves $K_{\Theta}$ invariant. Then by Theorem 6.6, for every conjugation $J$ in $\mathcal{H}$ such that $\Theta$ is $J$-symmetric, there exists a unitary operator $V_{0} \in L(\mathcal{H})$ such that $\mathbf{C}=M_{\mathbf{V}} \mathbf{C}_{\Theta, J}$, where $\mathbf{V}(z)=V_{0}$ 
a.e. on $\mathbb{T}$. Therefore, for two such conjugations $J$ and $J^{\prime}$ in $\mathcal{H}$ there exist unitary operators $V_{0}, V_{0}^{\prime}$ and constant operator valued functions $\mathbf{V}(z)=V_{0}$, $\mathbf{V}^{\prime}(z)=V_{0}^{\prime}($ a.e. on $\mathbb{T})$ such that

$$
\mathbf{C}=M_{\mathbf{V}} \mathbf{C}_{\Theta, J}=M_{\mathbf{V}^{\prime}} \mathbf{C}_{\Theta, J^{\prime}}
$$

Moreover, $V_{0}^{\prime}=V_{0} J J^{\prime}$.

Example 6.8. To illustrate Theorem 6.6 consider $\mathcal{H}=\mathbb{C}^{2}$ and the conjugation $J_{1}\left(z_{1}, z_{2}\right)=\left(\bar{z}_{1}, \bar{z}_{2}\right)$. Note that $\Theta(z)=\left[\begin{array}{cc}z & 0 \\ 0 & z^{2}\end{array}\right]$ is $J_{1}$-symmetric and defines a pure inner matrix valued function. Then $K_{\Theta}=\left\{\left(a_{0}, b_{0}+b_{1} z\right): a_{0}, b_{0}, b_{1} \in \mathbb{C}\right\}$ and the conjugation $\mathbf{C}_{\Theta, J_{1}}$ is equal to $\left[\begin{array}{cc}\tilde{J} & 0 \\ 0 & z \tilde{J}\end{array}\right]$.

Assume that $\mathbf{C}$ is an $\mathbf{M}_{z}$-conjugation. By Theorem 2.5

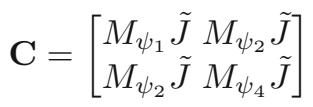

with $\psi_{i}$ satisfying (2.9)-(2.10). Hence

$$
\mathbf{C}\left(a_{0}, b_{0}+b_{1} z\right)=\left(\psi_{1} \bar{a}_{0}+\psi_{2}\left(\bar{b}_{0}+\bar{b}_{1} \bar{z}\right), \psi_{2} \bar{a}_{0}+\psi_{4}\left(\bar{b}_{0}+\bar{b}_{1} \bar{z}\right)\right)
$$

for any $a_{0}, b_{0}, b_{1} \in \mathbb{C}$. If $\mathbf{C}$ leaves subspace $K_{\Theta}$ invariant, then $\psi_{1}=\lambda_{1}$, $\psi_{2}=0, \psi_{4}=\lambda_{4} z$ with $\lambda_{i} \in \mathbb{T}$. It is clear that $\mathbf{C}=\left[\begin{array}{cc}\lambda_{1} & 0 \\ 0 & \lambda_{4}\end{array}\right] \mathbf{C}_{\Theta, J_{1}}$.

\section{Conjugations Between Model Spaces}

In this section we consider conjugations which map one model space into another. Let $\operatorname{dim} \mathcal{H}<\infty$.

Lemma 7.1. Let $\Theta, \Lambda \in H^{\infty}(L(\mathcal{H}))$ be two inner functions. Then the following are equivalent:
1. $\Lambda^{*} \Theta \in H^{\infty}(L(\mathcal{H}))$;
2. $\Theta H^{2}(\mathcal{H}) \subset \Lambda H^{2}(\mathcal{H})$;
3. $K_{\Lambda} \subset K_{\Theta}$.

Proof. If (1) holds, that is, $\Lambda^{*} \Theta=\Psi \in H^{\infty}(L(\mathcal{H}))$, then $\Theta=\Lambda \Psi$ and so (2) is satisfied. On the other hand, if (2) is satisfied, then by Lemma 6.2, $\Theta=\Lambda \Psi$ for some $\Psi \in H^{\infty}(L(\mathcal{H})), \Psi=\Lambda^{*} \Theta$ and (1) follows. The equivalence of (2) and (3) is obvious.

In view of Lemma 7.1 we will say that an operator valued inner function $\Lambda$ divides an operator valued inner function $\Theta$, and we write $\Lambda \leqslant \Theta$, if $\Lambda^{*} \Theta \in H^{\infty}(L(\mathcal{H}))$. Equivalently, $\Lambda \leqslant \Theta$, if $\Theta=\Lambda \Psi$ for some $\Psi \in H^{\infty}(L(\mathcal{H}))$ (clearly $\Psi=\Lambda^{*} \Theta$ is also an operator valued inner function).

Proposition 7.2. Let $\Theta, \Lambda \in H^{\infty}(L(\mathcal{H}))$ be two inner functions. If there exists a conjugation $J$ in $\mathcal{H}$ such that both $\Theta$ and $\Lambda$ are $J$-symmetric, then the following are equivalent:

1. $\Lambda^{*} \Theta \in H^{\infty}(L(\mathcal{H}))$; 
2. $\Theta \Lambda^{*} \in H^{\infty}(L(\mathcal{H}))$.

Proof. Assume that $\Theta \Lambda^{*} \in H^{\infty}(L(\mathcal{H}))$. Then $\Theta \Lambda^{*}\left(H^{2}(\mathcal{H})\right) \subset H^{2}(\mathcal{H})$. Note also that $\Theta \Lambda^{*}\left(\Lambda H^{2}(\mathcal{H})\right)=\Theta H^{2}(\mathcal{H})$ and

$$
\mathbf{C}_{\Theta, J}=M_{\Theta \Lambda^{*}} \mathbf{C}_{\Lambda, J} .
$$

Since both $\Lambda$ and $\Theta$ are $J$-symmetric, we have $\mathbf{C}_{\Lambda, J}\left(K_{\Lambda}\right)=K_{\Lambda}$ and $\mathbf{C}_{\Theta, J}\left(K_{\Theta}\right)=K_{\Theta}$. Hence

$$
\mathbf{C}_{\Theta, J}\left(K_{\Lambda}\right)=M_{\Theta \Lambda^{*}} \mathbf{C}_{\Lambda, J}\left(K_{\Lambda}\right)=M_{\Theta \Lambda^{*}}\left(K_{\Lambda}\right) \subset K_{\Theta},
$$

which implies that

$$
K_{\Lambda} \subset \mathbf{C}_{\Theta, J}\left(K_{\Theta}\right)=K_{\Theta} .
$$

By Lemma 7.1 it follows that $\Lambda^{*} \Theta \in H^{\infty}(L(\mathcal{H}))$. The other implication can be proved analogously.

Theorem 7.3. Let $\Theta, \Lambda \in H^{\infty}(L(\mathcal{H}))$ be two pure inner functions and assume that there exist conjugations $J_{\Theta}, J_{\Lambda}$ in $\mathcal{H}$ such that $\Theta$ is $J_{\Theta}$-symmetric and $\Lambda$ is $J_{\Lambda}$-symmetric. Moreover, let $\mathbf{C}$ be an $\mathbf{M}_{z}$-conjugation in $L^{2}(\mathcal{H})$. Then $\mathbf{C}\left(K_{\Lambda}\right) \subset K_{\Theta}$ if and only if $\mathbf{C}=\mathbf{C}_{\Gamma, J}$ for some inner function $\Gamma \in H^{\infty}(L(\mathcal{H}))$ and for some conjugation $J$ in $\mathcal{H}$ such that $\Lambda \leqslant \Gamma \leqslant \Theta$ and $\Gamma$ is J-symmetric. In particular, then $\Lambda \leqslant \Theta$.

Proof. Assume first that $\mathbf{C}=\mathbf{C}_{\Gamma, J}, \Gamma$ is $J$-symmetric and $\Lambda \leqslant \Gamma \leqslant \Theta$. Then $K_{\Lambda} \subset K_{\Gamma} \subset K_{\Theta}$ and

$$
\mathbf{C}\left(K_{\Lambda}\right)=\mathbf{C}_{\Gamma, J}\left(K_{\Lambda}\right) \subset \mathbf{C}_{\Gamma, J}\left(K_{\Gamma}\right)=K_{\Gamma} \subset K_{\Theta} .
$$

Assume now that $\mathbf{C}$ is an $\mathbf{M}_{z}$-conjugation such that $\mathbf{C}\left(K_{\Lambda}\right) \subset K_{\Theta}$. By Theorem 4.8 there exist $\mathbf{U}_{\Lambda}, \mathbf{U}_{\Theta} \in L^{\infty}(\mathcal{H})$ such that

$$
\mathbf{C}=M_{\mathbf{U}_{\Lambda}} \widetilde{\mathbf{J}}_{\Lambda}=\widetilde{\mathbf{J}}_{\Theta} M_{\mathbf{U}_{\Theta}^{*}} \text {, }
$$

the function $\mathbf{U}_{\Lambda}$ is unitary valued and $J_{\Lambda}$-symmetric, and the function $\mathbf{U}_{\Theta}$ is unitary valued and $J_{\Theta}$-symmetric. Moreover, it follows from Remark 4.9 that

$$
\mathbf{U}_{\Theta}(z)=\mathbf{U}_{\Lambda}(z) J_{\Lambda} J_{\Theta} \quad \text { a.e. on } \mathbb{T} \text {. }
$$

By Lemma 6.5 , for $x \in \mathcal{H}$ we have a.e. on $\mathbb{T}$,

$$
\begin{aligned}
\left(\mathbf{C C}_{\Lambda, J_{\Lambda}} k_{0}^{\Lambda} x\right)(z) & =\mathbf{U}_{\Lambda}(z) J_{\Lambda}\left(\left(\mathbf{C}_{\Lambda, J_{\Lambda}} k_{0}^{\Lambda} x\right)(z)\right) \\
& =\mathbf{U}_{\Lambda}(z) J_{\Lambda}\left(\bar{z}(\Lambda(z)-\Lambda(0)) J_{\Lambda} x\right) \\
& =\mathbf{U}_{\Lambda}(z) z\left(\Lambda(z)^{*}-\Lambda(0)^{*}\right) x \\
& =\mathbf{U}_{\Lambda}(z) z \Lambda(z)^{*}\left(1-\Lambda(z) \Lambda(0)^{*}\right) x .
\end{aligned}
$$

Define

$$
\mathbf{V}_{1}(z)=\mathbf{U}_{\Lambda}(z) z \Lambda(z)^{*} \quad \text { and } \quad \mathbf{W}_{1}(z)=\mathbf{V}_{1}(z)\left(1-\Lambda(z) \Lambda(0)^{*}\right)
$$

(a.e. on $\mathbb{T}$ ). Clearly, $\mathbf{V}_{1}, \mathbf{W}_{1} \in L^{\infty}(L(\mathcal{H}))$. By the calculations above, $M_{\mathbf{W}_{1}}\left(x e_{0}\right)=\mathbf{C C}_{\Lambda, J_{\Lambda}} k_{0}^{\Lambda} x \in H^{2}(\mathcal{H})$. Since $M_{\mathbf{W}_{1}}$ commutes with $\mathbf{M}_{z}$, we also get $M_{\mathbf{W}_{1}}\left(x e_{n}\right) \in H^{2}(\mathcal{H})$ for $n=0,1, \ldots$ Hence $M_{\mathbf{W}_{1}}\left(H^{2}(\mathcal{H})\right) \subset H^{2}(\mathcal{H})$ and $\mathbf{W}_{1} \in H^{\infty}(L(\mathcal{H}))$. 
Recalling that $\Lambda$ is a pure analytic function, we have that $z \mapsto(1-$ $\left.\Lambda(z) \Lambda(0)^{*}\right)^{-1}$ belongs to $H^{\infty}(L(\mathcal{H}))$. Hence also $\mathbf{V}_{1} \in H^{\infty}(L(\mathcal{H}))$ and $\mathbf{V}_{1}$ is inner, since its values are unitary operators.

On the other hand, using (7.1) we get (a.e. on $\mathbb{T}$ )

$$
\begin{aligned}
\left(\mathbf{C}_{\Theta, J_{\Theta}} \mathbf{C} k_{0}^{\Lambda} x\right)(z) & =\left(\mathbf{C}_{\Theta, J_{\Theta}} \widetilde{\mathbf{J}}_{\Theta} M_{\mathbf{U}_{\Theta}^{*}} k_{0}^{\Lambda} x\right)(z) \\
& =\Theta(z) \bar{z} \mathbf{U}_{\Theta}(z)^{*}\left(1-\Lambda(z) \Lambda(0)^{*}\right) x \\
& =\Theta(z) \bar{z} J_{\Theta} J_{\Lambda} \mathbf{U}_{\Lambda}(z)^{*}\left(1-\Lambda(z) \Lambda(0)^{*}\right) x .
\end{aligned}
$$

Define

$$
\mathbf{V}_{2}(z)=\Theta(z) \bar{z} J_{\Theta} J_{\Lambda} \mathbf{U}_{\Lambda}(z)^{*} \quad \text { and } \quad \mathbf{W}_{2}(z)=\mathbf{V}_{2}(z)\left(1-\Lambda(z) \Lambda(0)^{*}\right)
$$

(a.e. on $\mathbb{T}$ ). As above $H^{2}(\mathcal{H})$ is $M_{\mathbf{W}_{2}}$-invariant so $\mathbf{W}_{2} \in H^{\infty}(L(\mathcal{H})$ ), and consequently $\mathbf{V}_{2} \in H^{\infty}(L(\mathcal{H}))$ is an inner function. Since

$$
\mathbf{V}_{2}(z)=J_{\Theta} \Theta(z)^{*} z \mathbf{U}_{\Lambda}(z) J_{\Lambda} \quad \text { a.e. on } \mathbb{T},
$$

it follows from Lemma 6.1 that $\mathbf{V}_{3}(z)=\mathbf{U}_{\Lambda}(z)^{*} \bar{z} \Theta(z)$ (a.e. on $\mathbb{T}$ ) is also an inner function.

Define

$$
\Gamma(z)=\mathbf{U}_{\Lambda}(z) z \quad \text { a.e. on } \mathbb{T} \text {. }
$$

Then $\mathbf{V}_{1}=\Gamma \Lambda^{*} \in H^{\infty}(L(\mathcal{H}))$, and so $\Gamma=\mathbf{V}_{1} \Lambda$ is an inner function. Observe also that $\Gamma$ is $J_{\Lambda}$-symmetric. By Proposition 7.2 we also have $\Lambda^{*} \Gamma \in H^{\infty}(L(\mathcal{H}))$, and so $\Lambda \leqslant \Gamma$.

Moreover, $\mathbf{V}_{3}=\Gamma^{*} \Theta \in H^{\infty}(L(\mathcal{H}))$ and $\Gamma \leqslant \Theta$. In particular,

$$
\Lambda^{*} \Theta=\Lambda^{*} \Gamma \Gamma^{*} \Theta \in H^{\infty}(L(\mathcal{H})),
$$

that is, $\Lambda \leqslant \Theta$. Finally, a.e. on $\mathbb{T}$,

$$
(\mathbf{C} f)(z)=\mathbf{U}_{\Lambda}(z) J_{\Lambda}(f(z))=\Gamma(z) \bar{z} J_{\Lambda}(f(z))=\left(\mathbf{C}_{\Gamma, J_{\Lambda}} f\right)(z) .
$$

Remark 7.4. Note that in the proof of Theorem 7.3 we actually did not use the fact that $\Theta$ is pure. Moreover, if we assume that $\Lambda$ and $\Theta$ are $J$-symmetric, it follows from the proof that $\Gamma$ can be chosen to be $J$-symmetric as well.

Remark 7.5. Let $\mathbf{C}=\mathbf{C}_{\Gamma, J}$ for an inner function $\Gamma \in H^{\infty}(L(\mathcal{H}))$, which is $J$-symmetric and such that $\Lambda \leqslant \Gamma \leqslant \Theta$. Then for any conjugation $J^{\prime}$ in $\mathcal{H}$ the function $\Gamma^{\prime}$ defined by

$$
\Gamma^{\prime}(z)=\Gamma(z) J J^{\prime} \quad \text { a.e. on } \mathbb{T}
$$

is $J^{\prime}$-symmetric, $\Lambda \leqslant \Gamma^{\prime} \leqslant \Theta$ and $\mathbf{C}=\mathbf{C}_{\Gamma^{\prime}, J^{\prime}}$.

Remark 7.6. If $\Lambda=\Theta$ is $J$-symmetric and $\mathbf{C}$ is an $\mathbf{M}_{z}$-conjugation in $L^{2}(\mathcal{H})$ such that $\mathbf{C}\left(K_{\Theta}\right) \subset K_{\Theta}$, then by Theorem 7.3 and Remark 7.4 there exists an inner $J$-symmetric function $\Gamma$ such that $\mathbf{C}=\mathbf{C}_{\Gamma, J}$ and $\Theta \leqslant \Gamma \leqslant \Theta$. The last condition implies that $\mathbf{V}=\Gamma \Theta^{*}$ is a unitary constant and a.e. on $\mathbb{T}$,

$$
(\mathbf{C} f)(z)=\Gamma(z) \bar{z} J(f(z))=\Gamma(z) \Theta(z)^{*} \Theta(z) \bar{z} J(f(z))=\left(M_{\mathbf{V}} \mathbf{C}_{\Theta, J} f\right)(z) .
$$

Moreover, $\mathbf{V}$ satisfies (6.2) and $M_{\mathbf{V}}$ is $\mathbf{C}_{\Theta, J}$-symmetric.

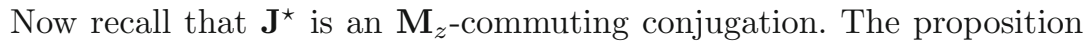
below shows some basic properties of $\mathbf{J}^{\star}$ as to model spaces. 
Proposition 7.7. Let $J$ be a conjugation in $\mathcal{H}$ and let $\Theta$ be a J-symmetric inner function. Then

$$
\begin{aligned}
& \text { 1. } \mathbf{J}^{\star} M_{\Theta}=M_{\Theta \#} \mathbf{J}^{\star} ; \\
& \text { 2. } \mathbf{J}^{\star}\left(\Theta H^{2}(\mathcal{H})\right)=\Theta^{\#} H^{2}(\mathcal{H}) ; \\
& \text { 3. } \mathbf{J}^{\star}\left(K_{\Theta}\right)=K_{\Theta}, \\
& \text { 4. } \mathbf{J}^{\star}\left(k_{0}^{\Theta} x\right)=k_{0}^{\Theta \#} J x .
\end{aligned}
$$

In what follows we describe all $\mathbf{M}_{z}$-commuting conjugations mapping one model space into another.

Theorem 7.8. Let $\Theta, \Lambda \in H^{\infty}(L(\mathcal{H}))$ be two pure inner functions and assume that there exist conjugations $J_{\Theta}, J_{\Lambda}$ in $\mathcal{H}$ such that $\Theta$ is $J_{\Theta}$-symmetric and $\Lambda$ is $J_{\Lambda}$-symmetric. Assume that $\mathbf{C}$ is an $\mathbf{M}_{z}$-commuting conjugation in $L^{2}(\mathcal{H})$. Then $\mathbf{C}\left(K_{\Lambda}\right) \subset K_{\Theta}$ if and only if there is a unitary $J_{\Lambda}$-symmetric operator $U_{0} \in L(\mathcal{H})$ such that $\mathbf{C}=M_{\mathbf{U}_{\Lambda}} \mathbf{J}_{\Lambda}^{\star}$, where $\mathbf{U}_{\Lambda}$ is a constant operator valued function, $\mathbf{U}_{\Lambda}(z)=U_{0}$ for almost all $z \in \mathbb{T}$ and $U_{0} \Lambda^{\#} \leqslant \Theta$.

Proof. Since $\mathbf{C}$ is $\mathbf{M}_{z}$-commuting, then by Theorem 4.3 there is $\mathbf{U}_{\Lambda} \in$ $L^{\infty}(L(\mathcal{H}))$ such that $\mathbf{U}_{\Lambda}(z)$ is a unitary operator for almost all $z \in \mathbb{T}, M_{\mathbf{U}_{\Lambda}}$ is $\mathbf{J}_{\Lambda}^{\star}$-symmetric and $\mathbf{C}=M_{\mathbf{U}_{\Lambda}} \mathbf{J}_{\Lambda}^{\star}$. Note that using Lemma 6.5 and Proposition 7.7 (4) a.e. on $\mathbb{T}$ we have

$$
\begin{aligned}
\left(\mathbf{C C}_{\Lambda, J_{\Lambda}} \widetilde{k_{0}^{\Lambda}} x\right)(z) & =\mathbf{U}_{\Lambda}(z) \mathbf{J}_{\Lambda}^{\star}\left(k_{0}^{\Lambda} J_{\Lambda} x\right)(z) \\
& =\mathbf{U}_{\Lambda}(z)\left(1-\Lambda^{\#}(z)\left(\Lambda^{\#}(0)\right)^{*}\right) x .
\end{aligned}
$$

Define $\mathbf{V}(z)=\mathbf{U}_{\Lambda}(z)\left(1-\Lambda^{\#}(z)\left(\Lambda^{\#}(0)\right)^{*}\right)$ a.e. on $\mathbb{T}$. It is clear that $\mathbf{V} \in$ $L^{\infty}(L(\mathcal{H}))$ and by $(7.2)$ we have that $M_{\mathbf{V}}\left(x e_{0}\right)=\mathbf{C C}_{\Lambda, J_{\Lambda}} \widetilde{k_{0}^{\Lambda}} x \in H^{2}(\mathcal{H})$ because $\mathbf{C}\left(K_{\Lambda}\right) \subset K_{\Theta}$. Since $M_{\mathbf{V}}$ commutes with $\mathbf{M}_{z}$, we have also that $M_{\mathbf{V}}\left(x e_{n}\right) \in H^{2}(\mathcal{H})$ for $n=1,2, \ldots$ Hence $M_{\mathbf{V}}\left(H^{2}(\mathcal{H})\right) \subset H^{2}(\mathcal{H})$ and $\mathbf{V} \in$ $H^{\infty}(L(\mathcal{H}))$. Since $\Lambda$ is pure, it follows that $z \mapsto\left(1-\Lambda^{\#}(z)\left(\Lambda^{\#}(0)\right)^{*}\right)^{-1} \in$ $H^{\infty}(L(\mathcal{H}))$. By $(7.2)$ we obtain that

$$
\mathbf{U}_{\Lambda}=\mathbf{V}\left(1-\Lambda^{\#}\left(\Lambda^{\#}(0)\right)^{*}\right)^{-1} \in H^{\infty}(L(\mathcal{H})),
$$

which implies that $\mathbf{C}$ leaves $H^{2}(\mathcal{H})$ invariant. Applying Theorem 5.1 we get that $\mathbf{U}_{\Lambda}$ is a constant operator valued function and $\mathbf{U}_{\Lambda}(z)=U_{0}$ a.e. on $\mathbb{T}$. Since $M_{\mathbf{U}_{\Lambda}}$ is $\mathbf{J}_{\Lambda}^{\star}$-symmetric thus $U_{0}$ is $J$-symmetric by Proposition 4.2.

Note that by Proposition 7.7 (3), we have

$$
\mathbf{C}\left(K_{\Lambda}\right)=U_{0} K_{\Lambda \#} .
$$

Since $U_{0}$ is unitary, then $U_{0} K_{\Lambda^{\#}}=K_{U_{0} \Lambda^{\#}}$. Hence by Lemma 7.1,

$$
\mathbf{C}\left(K_{\Lambda}\right) \subset K_{\Theta}
$$

if and only if $U_{0} \Lambda^{\#} \leqslant \Theta$.

Remark 7.9. Consider a pure inner function $\Theta \in H^{\infty}(L(\mathcal{H}))$ and a conjugation $J_{\Theta}$ in $\mathcal{H}$ such that $\Theta$ is $J_{\Theta}$-symmetric. As a consequence of Theorem 7.8 we have that if $\mathbf{C}$ is an $\mathbf{M}_{z}$-commuting conjugation in $L^{2}(\mathcal{H})$, then $\mathbf{C}\left(K_{\Theta}\right) \subset K_{\Theta}$ if and only if there is a unitary operator $U_{0} \in L(\mathcal{H})$ such that $\mathbf{C}=M_{\mathbf{U}_{\Theta}} \mathbf{J}_{\Theta}^{\star}$, where $\mathbf{U}_{\Theta}$ is a constant $J_{\Theta}$-symmetric operator valued 
function, $\mathbf{U}_{\Theta}(z)=U_{0}$ for almost all $z \in \mathbb{T}$ and $U_{0} \Theta^{\#} \leqslant \Theta^{\#}$. Note that if $U_{0}$ commutes with $\Theta$, the last condition is always satisfied, since then $\left(U_{0} \Theta^{\#}\right)^{*} \Theta^{\#}=U_{0}^{*} \in H^{\infty}(L(\mathcal{H}))$.

\section{Conjugations and Shift Invariant Subspaces}

Let $\operatorname{dim} \mathcal{H}<\infty$ and let $\Theta, \Lambda \in H^{\infty}(L(\mathcal{H}))$ be two inner functions. For any fixed conjugation $J$ in $\mathcal{H}$ define

$$
\mathbf{C}_{J}^{\Lambda, \Theta}=M_{\Theta} \mathbf{J}^{\star} M_{\Lambda^{*}} .
$$

Clearly, $\mathbf{C}_{J}^{\Lambda, \Theta}$ is an antilinear isometry. Moreover, it is easy to see that $\mathbf{C}_{J}^{\Lambda, \Theta}$ maps $\Lambda H^{2}(\mathcal{H})$ onto $\Theta H^{2}(\mathcal{H})$.

Proposition 8.1. The antilinear operator $\mathbf{C}_{J}^{\Lambda, \Theta}$ is an involution (and hence a conjugation in $L^{2}(\mathcal{H})$ ) if and only if

$$
\Theta(z) J \Lambda^{\#}(z)=\Lambda(z) J \Theta^{\#}(z) \quad \text { a.e. on } \mathbb{T} \text {. }
$$

Proof. We have

$$
\left(\mathbf{C}_{J}^{\Lambda, \Theta}\right)^{2}=M_{\Theta} \mathbf{J}^{\star} M_{\Lambda^{*}} M_{\Theta} \mathbf{J}^{\star} M_{\Lambda^{*}}=I_{L^{2}(\mathcal{H})}
$$

if and only if

$$
M_{\Theta} \mathbf{J}^{\star} M_{\Lambda^{*}}=M_{\Lambda} \mathbf{J}^{\star} M_{\Theta^{*}} .
$$

Since for $\mathbf{f} \in L^{2}(\mathcal{H})$ a.e. on $\mathbb{T}$,

$$
\left(M_{\Theta} \mathbf{J}^{\star} M_{\Lambda^{*}} \mathbf{f}\right)(z)=\Theta(z) J\left(\Lambda(\bar{z})^{*} \mathbf{f}(\bar{z})\right)=\left(M_{\Theta J \Lambda} \widetilde{\mathbf{J}} \mathbf{J}^{\star} \mathbf{f}\right)(z)
$$

and

$$
\left(M_{\Lambda} \mathbf{J}^{\star} M_{\Theta *} \mathbf{f}\right)(z)=\Lambda(z) J\left(\Theta(\bar{z})^{*} \mathbf{f}(\bar{z})\right)=\left(M_{\Lambda J \Theta \#} \widetilde{\mathbf{J}}{ }^{\star} \mathbf{f}\right)(z),
$$

we see that (8.2) is equivalent to (8.1).

For $\mathbf{F} \in L^{\infty}(L(\mathcal{H}))$ and a conjugation $J$ in $\mathcal{H}$ define

$$
\mathbf{F}_{J}(z)=J \mathbf{F}(\bar{z}) J, \quad \text { a.e. on } \mathbb{T} \text {. }
$$

Clearly, $\mathbf{F}_{J} \in L^{\infty}(L(\mathcal{H}))$. Moreover, $\mathbf{F}_{J} \in H^{\infty}(L(\mathcal{H}))$ if and only if $\mathbf{F} \in$ $H^{\infty}(L(\mathcal{H}))$ (see the proof of Lemma 6.1), and $\mathbf{F}_{J}$ is an inner function if and only if $\mathbf{F}$ is. It is easy to verify the following.

Lemma 8.2. Let $\mathbf{F}, \mathbf{G} \in L^{\infty}(L(\mathcal{H}))$ and let $J$ be a conjugation in $\mathcal{H}$. Then

1. $\left(\mathbf{F}_{J}\right)_{J}=\mathbf{F}$;

2. $(\mathbf{F G})_{J}=\mathbf{F}_{J} \mathbf{G}_{J}$;

3. $\left(\mathbf{F}_{J}\right)^{*}(z)=J \mathbf{F}^{\#}(z) J=\left(\mathbf{F}^{*}\right)_{J}(z)$ a.e. on $\mathbb{T}$;

4. $\left(\mathbf{F}_{J}\right)^{\#}(z)=J \mathbf{F}^{*}(z) J=\left(\mathbf{F}^{\#}\right)_{J}(z)$ a.e. on $\mathbb{T}$.

Moreover, from the proof of Proposition 4.2 we get:

Lemma 8.3. Let $\mathbf{F} \in L^{\infty}(L(\mathcal{H}))$ and let $J$ be a conjugation in $\mathcal{H}$. Then

$$
\mathbf{J}^{\star} M_{\mathbf{F}} \mathbf{J}^{\star}=M_{\mathbf{F}_{J}} .
$$


Remark 8.4. Note that condition (8.1) can be expressed as

$$
\Theta \Lambda_{J}^{*}=\Lambda \Theta_{J}^{*}
$$

Indeed, (8.1) is equivalent to

$$
\Theta(z) J \Lambda^{\#}(z) J=\Lambda(z) J \Theta^{\#}(z) J \quad \text { a.e. on } \mathbb{T},
$$

and by Lemma $8.2(3)$, for almost all $z \in \mathbb{T}, J \Lambda^{\#}(z) J=\Lambda_{J}^{*}(z)$ and $J \Theta^{\#}(z) J=\Theta_{J}^{*}(z)$.

Remark 8.5. If $\mathcal{H}=\mathbb{C}$ and $J(w)=\bar{w}, w \in \mathbb{C}$, then for $\varphi \in L^{\infty}(\mathbb{T})$ we have

$$
\varphi_{J}=\varphi^{\#} \text {. }
$$

Moreover, for scalar inner functions $\theta$ and $\alpha$ condition (8.1) (or (8.3)) takes form

$$
\theta(z) \alpha(\bar{z})=\alpha(z) \theta(\bar{z}) \quad \text { a.e. on } \mathbb{T},
$$

which in this case is equivalent to $\theta \theta^{\#}=\alpha \alpha^{\#}$ (see [2, Theorem 5.2]).

Theorem 8.6. Let $\Theta, \Lambda \in H^{\infty}(L(\mathcal{H}))$ be two inner functions and let $J$ be a conjugation in $\mathcal{H}$. There exists an $\mathbf{M}_{z}$-commuting conjugation $\mathbf{C}$ in $L^{2}(\mathcal{H})$ such that $\mathbf{C}\left(\Lambda H^{2}(\mathcal{H})\right) \subset \Theta H^{2}(\mathcal{H})$ if and only if there is an inner function $\Psi \in H^{\infty}(L(\mathcal{H}))$ such that

$$
\left(\Psi \Lambda^{*} \Theta\right)_{J}=\left(\Psi \Lambda^{*} \Theta\right)^{*} .
$$

In that case, $\mathbf{C}=\mathbf{C}_{J}^{\Lambda, \Gamma}$ for some inner function $\Gamma \in H^{\infty}(L(\mathcal{H}))$ such that $\Theta \leqslant \Gamma$ and

$$
\Gamma \Lambda_{J}^{*}=\Lambda \Gamma_{J}^{*}
$$

Assume at first that there exists an inner function $\Psi \in H^{\infty}(L(\mathcal{H}))$ such that (8.4) holds. Then

$$
\Theta^{*} \Lambda \Psi^{*}=\left(\Psi \Lambda^{*} \Theta\right)^{*}=\left(\Psi \Lambda^{*} \Theta\right)_{J}=\Psi_{J} \Lambda_{J}^{*} \Theta_{J},
$$

and so

$$
\Lambda \Psi^{*} \Theta_{J}^{*}=\Theta \Psi_{J} \Lambda_{J}^{*}
$$

Put $\Gamma=\Theta \Psi_{J}$. Then $\Gamma \in H^{\infty}(L(\mathcal{H}))$ is an inner function, $\Theta \leqslant \Gamma$ and by $(8.6)$,

$$
\Lambda \Gamma_{J}^{*}=\Lambda\left(\Theta_{J} \Psi\right)^{*}=\Lambda \Psi^{*} \Theta_{J}^{*}=\Theta \Psi_{J} \Lambda_{J}^{*}=\Gamma \Lambda_{J}^{*} .
$$

Thus (8.5) holds and, by Proposition 8.1 and Remark 8.4, $\mathbf{C}=\mathbf{C}_{J}^{\Lambda, \Gamma}$ is a conjugation in $L^{2}(\mathcal{H})$. Moreover, it is an $\mathbf{M}_{z}$-commuting conjugation such that

$$
\mathbf{C}\left(\Lambda H^{2}(\mathcal{H})\right)=\Gamma H^{2}(\mathcal{H}) \subset \Theta H^{2}(\mathcal{H})
$$

Assume now that $\mathbf{C}$ is an $\mathbf{M}_{z}$-commuting conjugation in $L^{2}(\mathcal{H})$ such that $\mathbf{C}\left(\Lambda H^{2}(\mathcal{H})\right) \subset \Theta H^{2}(\mathcal{H})$. By Theorem 4.3 and Proposition $4.2(2)$ there exists a unitary valued $\mathbf{U} \in L^{\infty}(L(\mathcal{H}))$ such that

$$
\mathbf{C}=M_{\mathbf{U}} \mathbf{J}^{\star}=\mathbf{J}^{\star} M_{\mathbf{U}^{*}}
$$


and $J \mathbf{U}(z) J=\mathbf{U}^{\#}(z)$ a.e. on $\mathbb{T}$. Therefore,

$$
\mathbf{C}\left(\Lambda H^{2}(\mathcal{H})\right)=\mathbf{J}^{\star} M_{\mathbf{U}^{*}} M_{\Lambda}\left(H^{2}(\mathcal{H})\right) \subset M_{\Theta}\left(H^{2}(\mathcal{H})\right),
$$

and by Lemma 8.3,

$$
M_{\mathbf{U}^{*} \Lambda}\left(H^{2}(\mathcal{H})\right) \subset \mathbf{J}^{\star} M_{\Theta}\left(H^{2}(\mathcal{H})\right)=M_{\Theta_{J}} \mathbf{J}^{\star}\left(H^{2}(\mathcal{H})\right)=\Theta_{J} H^{2}(\mathcal{H}) .
$$

By Lemma 6.2, $\mathbf{U}^{*} \Lambda \in H^{\infty}(L(\mathcal{H}))$ and there exists an inner function $\Psi \in$ $H^{\infty}(L(\mathcal{H}))$ such that $\mathbf{U}^{*} \Lambda=\Theta_{J} \Psi$. Note that by the fact that $J \mathbf{U}(z) J=$ $\mathbf{U}^{\#}(z)$ a.e. on $\mathbb{T}$ and Lemma $8.2(3)$, we get $\mathbf{U}_{J}^{*}=\mathbf{U}$. It follows that

$$
\Theta \Psi_{J} \Lambda_{J}^{*}=\left(\Theta_{J} \Psi \Lambda^{*}\right)_{J}=\left(\mathbf{U}^{*} \Lambda \Lambda^{*}\right)_{J}=\mathbf{U}=\Lambda \Psi^{*} \Theta_{J}^{*},
$$

which is an equivalent form of (8.4). Moreover, the above means that the function $\Gamma=\Theta \Psi_{J}=\mathbf{U}_{J}^{*} \Lambda_{J}$ satisfies (8.5) (since $\Gamma_{J}^{*}=\Psi^{*} \Theta_{J}^{*}$ ). Clearly, $\Gamma \in H^{\infty}(L(\mathcal{H}))$ is an inner function and $\Theta \leqslant \Gamma$. Moreover,

$\mathbf{C}=M_{\mathbf{U}} \mathbf{J}^{\star}=M_{\mathbf{U}_{J}^{*}} \mathbf{J}^{\star} M_{\Lambda} M_{\Lambda^{*}}=M_{\mathbf{U}_{J}^{*} \Lambda_{J}} \mathbf{J}^{\star} M_{\Lambda^{*}}=M_{\Gamma} \mathbf{J}^{\star} M_{\Lambda^{*}}=\mathbf{C}_{J}^{\Lambda, \Gamma}$.

Remark 8.7. Let $\Theta \in H^{\infty}(L(\mathcal{H}))$ be an inner function. Assume that $\mathbf{C}$ is an $\mathbf{M}_{z}$-commuting conjugation in $L^{2}(\mathcal{H})$ such that $\mathbf{C}\left(\Theta H^{2}(\mathcal{H})\right) \subset \Theta H^{2}(\mathcal{H})$. By Theorem 8.6 we obtain that $\mathbf{C}=\mathbf{C}_{J}^{\Theta, \Gamma}$ for some inner function $\Gamma \in$ $H^{\infty}(L(\mathcal{H}))$ such that $\Theta \leqslant \Gamma$ and

$$
\Gamma \Theta_{J}^{*}=\Theta \Gamma_{J}^{*} .
$$

Therefore there exists $\Psi \in H^{\infty}(L(\mathcal{H}))$ such that $\Gamma=\Theta \Psi$ and by (8.7),

$$
\Theta \Psi \Theta_{J}^{*}=\Theta \Psi_{J}^{*} \Theta_{J}^{*} \text {. }
$$

It follows that $\Psi=\Psi_{J}^{*}$. Since $\Psi_{J}, \Psi_{J}^{*} \in H^{\infty}(L(\mathcal{H}))$, so $\Psi$ must be a unitary constant. Assume that $\Psi(z)=U_{0} \in L(\mathcal{H})$ a.e. on $\mathbb{T}$, then $\Gamma(z)=\Theta(z) U_{0}$ a.e. on $\mathbb{T}$ and

$$
\mathbf{C}=M_{\Gamma} \mathbf{J}^{\star} M_{\Theta^{*}}=M_{\Theta} M_{U_{0}} \mathbf{J}^{\star} M_{\Theta^{*}}=M_{\Theta U_{0} \Theta^{*}} M_{\Theta} \mathbf{J}^{\star} M_{\Theta^{*}} .
$$

Note that by (8.7) we now have

$$
\Theta(z) U_{0} J \Theta(\bar{z})^{*} J=\Theta(z) J U_{0}^{*} \Theta(\bar{z})^{*} J \text { a.e. on } \mathbb{T},
$$

which implies that $U_{0} J=J U_{0}^{*}$, i.e., $U_{0}$ is $J$-symmetric. Recalling the scalar case considered in [2, Corollary 5.4] one can expect that $\Theta U_{0} \Theta^{*}$ is a unitary constant. This is not necessarily true (see Example 8.8).

Example 8.8. Let $\mathcal{H}=\mathbb{C}^{2}$ and consider the conjugation $J\left(z_{1}, z_{2}\right)=\left(\bar{z}_{1}, \bar{z}_{2}\right)$ in $\mathbb{C}^{2}$. If we take $\Theta=\left[\begin{array}{ll}1 & 0 \\ 0 & z\end{array}\right]$ and $U_{0}=\left[\begin{array}{ll}0 & 1 \\ 1 & 0\end{array}\right]$. It is easy to see that both $\Theta$ and $U_{0}$ are $J$-symmetric, but $\Theta U_{0} \Theta^{*}=\left[\begin{array}{ll}0 & \bar{z} \\ z & 0\end{array}\right]$, so it is not constant. 
Open Access. This article is licensed under a Creative Commons Attribution 4.0 International License, which permits use, sharing, adaptation, distribution and reproduction in any medium or format, as long as you give appropriate credit to the original author(s) and the source, provide a link to the Creative Commons licence, and indicate if changes were made. The images or other third party material in this article are included in the article's Creative Commons licence, unless indicated otherwise in a credit line to the material. If material is not included in the article's Creative Commons licence and your intended use is not permitted by statutory regulation or exceeds the permitted use, you will need to obtain permission directly from the copyright holder. To view a copy of this licence, visit http:// creativecommons.org/licenses/by/4.0/.

Publisher's Note Springer Nature remains neutral with regard to jurisdictional claims in published maps and institutional affiliations.

\section{References}

[1] Bercovici, H.: Operator Theory and Arithmetic in $H^{\infty}$, Mathematical Surveys and Monographs, vol. 26. Amer. Math. Soc, Providence, RI (1988)

[2] Câmara, C., Kliś-Garlicka, K., Łanucha, B., Ptak, M.: Conjugations in $L^{2}$ and their invariants. Anal. Math. Phys. 10, 22 (2020). https://doi.org/10.1007/ s13324-020-00364-5

[3] Câmara, C., Kliś-Garlicka, K., Ptak, M.: Asymmetric truncated Toeplitz operators and conjugations. Filomat 33, 3697-3710 (2019)

[4] Chevrot, N., Fricain, E., Timotin, D.: The characteristic function of a complex symmetric contraction. Proc. Am. Math. Soc. 135, 2877-2886 (2007)

[5] Cima, J.A., Garcia, S.R., Ross, W.T., Wogen, W.R.: Truncated Toeplitz operators: spatial isomorphism, unitary equivalence, and similarity. Indiana Univ. Math. J. 59(2), 595-620 (2010)

[6] Garcia, S.R., Mashreghi, J., Ross, W.T.: Introduction to Model Spaces and Their Operators. Cambridge University Press, Cambridge (2016)

[7] Garcia, S.R., Putinar, M.: Complex symmetric operators and applications. Trans. Am. Math. Soc. 358, 1285-1315 (2006)

[8] Garcia, S.R., Prodan, E., Putinar, M.: Mathematical and physical aspects of complex symmetric operators. J. Phys A Math. Theor. 47, 353001 (2014)

[9] Garcia, S.R., Putinar, M.: Complex symmetric operators and applications II. Trans. Am. Math. Soc. 359, 3913-3931 (2007)

[10] Khan, R., Timotin, D.: Matrix valued trancated Toeplitz operators: basic properties. Complex Anal. Oper. Theory 12, 997-1014 (2018). https://doi.org/10. 1007/s11785-017-0675-3

[11] Ko, E., Lee, J.E.: Remark on complex symmetric operator matrices. Linear Multilinear Algebra (2018). https://doi.org/10.1080/03081087.2018.1450350

[12] Sz-Nagy, B., Foias, C.F., Bercovici, H., Kérchy, L.: Harmonic Analysis of Operators on a Hilbert Space, 2nd edn. Springer, London (2010)

[13] Radjavi, H., Rosenthal, P.: Invariant Subspaces. Springer, New York (1973)

[14] Sarason, D.: Algebraic properties of truncated Toeplitz operators. Oper. Matrices 1, 491-526 (2007) 


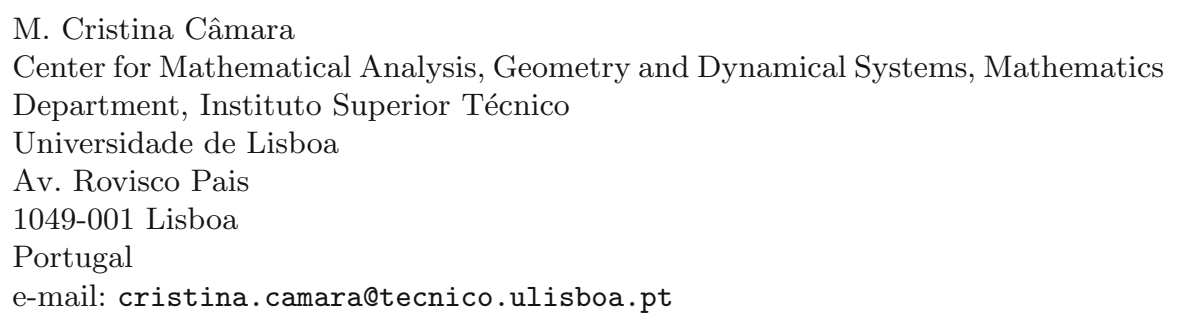

Kamila Kliś-Garlicka $(\bowtie)$ and Marek Ptak

Department of Applied Mathematics

University of Agriculture

ul. Balicka 253c

30-198 Kraków

Poland

e-mail: rmklis@cyfronet.pl

Marek Ptak

e-mail: rmptak@cyf-kr.edu.pl

Bartosz Łanucha

Department of Mathematics

Maria Curie-Skłodowska University

Maria Curie-Skłodowska Square 1

20-031 Lublin

Poland

e-mail: bartosz.lanucha@poczta.umcs.lublin.pl

Received: January 17, 2020.

Revised: September 9, 2020. 